\title{
BEING VAN KAMPEN IS A UNIVERSAL PROPERTY*
}

\author{
TOBIAS HEINDEL ${ }^{a}$ AND PAWEE SOBOCIŃSKI ${ }^{b}$ \\ ${ }^{a}$ Laboratoire d'Informatique de Paris-Nord, Université de Paris, France \\ e-mail address: Tobias.Heindel@lipn.univ-paris13.fr \\ ${ }^{b}$ DSSE, Electronics and Computer Science, University of Southampton, United Kingdom \\ e-mail address: ps@ecs.soton.ac.uk
}

\begin{abstract}
Colimits that satisfy the Van Kampen condition have interesting exactness properties. We show that the elementary presentation of the Van Kampen condition is actually a characterisation of a universal property in the associated bicategory of spans. The main theorem states that Van Kampen cocones are precisely those diagrams in a category that induce bicolimit diagrams in its associated bicategory of spans, provided that the category has pullbacks and enough colimits.
\end{abstract}

\section{INTRODUCTION}

Exactness, or in other words, the relationship between limits and colimits in various categories of interest is a research topic with several applications in theoretical computer science, including the solution of recursive domain equations [35], semantics of concurrent programming languages [37] and the study of formal grammars and transformation systems [10]. Researchers have identified several classes of categories in which certain limits and colimits relate to each other in useful ways; extensive categories [7, 31] and adhesive categories [29] are two relatively recent examples. Going further back, research on toposes and quasitoposes involved elaborate study of their exactness properties [20, 38].

Extensive categories [7 have coproducts that are "well-behaved" with respect to pullbacks; more concretely, they are disjoint and stable under pullback. Extensivity has been used by mathematicians [6] and computer scientists [33] alike. In the presence of products, extensive categories are distributive [7] and thus can be used, for instance, to model circuits [36] or to give models of specifications [18. Sets and topological spaces inhabit extensive categories while quasitoposes are not, in general, extensive [21.

Adhesive categories [28, 29] have pushouts along monos that are similarly "well-behaved" with respect to pullbacks - they are instances of Van Kampen squares. Adhesivity has been used as a categorical foundation for double-pushout graph transformation [28, 11] and has

1998 ACM Subject Classification: F.3.2, F.4.2.

Key words and phrases: category theory, extensive categories, adhesive categories, bicategories, bicolimits.

* This paper is an extended version of the CALCO '09 paper "Van Kampen colimits as bicolimits in Span" [17. 
found several related applications [12, 34]. Toposes are adhesive [30] but quasitoposes, in general, are not [22].

The elementary characterisations of coproducts in extensive categories and pushouts along monos in adhesive categories can be seen as specific instances of a general condition that can be expressed for any colimit. Cockett and Guo dubbed the colimits that satisfy this condition Van Kampen (VK) colimits [9], generalising the Van Kampen squares of [28]. Indeed, examples of VK-colimits include coproducts in extensive categories and pushouts along monos in adhesive categories; the simplest example is a strict initial object.

The definition of VK-colimits relies only on elementary notions of category theory. This feature, while attractive because of the implied simplicity, obscures relationships with other categorical concepts; the mathematical meaning of vK-colimits, so to speak. More abstract characterisations exist for extensive and adhesive categories. For instance, a category $\mathbf{C}$ is extensive if and only if the functor $+: \mathbf{C} \downarrow A \times \mathbf{C} \downarrow B \rightarrow \mathbf{C} \downarrow A+B$ is an equivalence for any $A, B \in \mathbf{C}$ [31, 7]; adhesive categories can be characterised in a similar manner [29]. Our definition of VK-cocone is of the latter kind, i.e. in terms of an equivalence of categories. We also provide an elementary characterisation in the spirit of Cockett and Guo.

This paper contains one central theorem: vK-cocones are those diagrams that are bicolimit diagrams when embedded in the associated bicategory of spans. Bicolimits are the canonical notion of colimit in a bicategory. This characterises "being Van Kampen" as a universal property. We believe that this insight captures and explains the essence of the various aforementioned well-behaved colimits studied in the literature.

Structure of the paper. In Section 1 we examine the category of spans and its applications. In Section 2 we exhibit the relationship between coproducts in extensive categories and coproducts in their associated categories of spans, and explain why it is necessary to consider bicategories of spans in order to extend this relationship to arbitrary colimits. In Section 3 we isolate the relevant class of bicategories and recall the related notions. In Section 4 we give a definition of VK-cocones together with an elementary characterisation and several examples. In Section 5 we recall the definition of bicolimits and prove several technical lemmas that allow us to pass between related concepts in a category and its associated bicategory of spans. The main theorem is proved in Section 6 .

\section{Spans AND Generalised RELATions}

There are several concepts in category theory that generalise relations between sets. The set theoretical concept of multirelation from $C$ to $D$ is a span of functions $C \leftarrow l-X-r \rightarrow D$, which we will denote $(l, X, r): C \rightarrow D$, or simply $(l, r)$. The set $X$ is sometimes referred to as the carrier. Roughly, a pair of elements $(c, d) \in C \times D$ can be related in a number of ways; concretely this is determined by the size of the preimage at $(c, d)$ of the function $\langle l, r\rangle: X \rightarrow C \times D$, i.e. $\langle l, r\rangle^{-1}(c, d)$. Two such spans $(l, X, r)$ and $\left(l^{\prime}, X^{\prime}, r^{\prime}\right)$ would normally be considered equivalent if there is a bijection $\varphi: X \rightarrow X^{\prime}$ that satisfies $l^{\prime} \varphi=l$ and $r^{\prime} \varphi=r$. The existence of such a bijection yields an equivalence relation; quotienting by this gives what we shall refer to as an abstract span. Sometimes, for emphasis, we shall refer to ordinary spans as concrete spans. A multirelation is thus an abstract span in the category of sets and functions. Ordinary relations are captured by those abstract spans in which $\langle l, r\rangle$ is injective. 
The concept of a span of morphisms makes sense in any category $\mathbf{C}$, not only Set; indeed we shall make use of the notation introduced in the previous paragraph generally. Extra structure is needed in order to compose spans, hence from this point onwards we assume that $\mathbf{C}$ has pullbacks. Below we give an overview of the category of $\mathbf{C}$-spans, denoted by $\mathrm{Sp}(\mathbf{C})$. Figure 1 accounts for its morphisms, identities and composition. More explicitly,
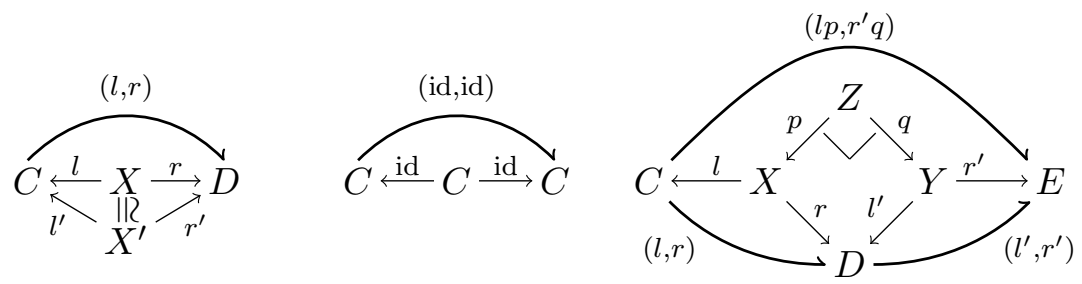

FiguRE 1. Abstract spans, identities and composition via pullback

$\operatorname{Sp}(\mathbf{C})$ has the same objects as $\mathbf{C}$ and a morphism from $C$ to $D$ is an equivalence class of spans $C \leftarrow l-X-r \rightarrow D$. The identity on an object $C$ is a span of identities in $\mathbf{C}$; composition is obtained via pullback as illustrated in Figure 1 on the right. It is not difficult to check that these definitions yield a category. Moreover, when $\mathbf{C}=$ Set this yields the expected composition of multirelations. Moreover, $\mathbf{C}$ has a standard embedding $\Gamma: \mathbf{C} \rightarrow \operatorname{Sp}(\mathbf{C})$ into the span category. This inclusion acts as the identity on objects and maps each morphism $f: A \rightarrow B$ in $\mathbf{C}$ to its graph $\Gamma(f: A \rightarrow B)=\left(\operatorname{id}_{A}, f\right)$; hence this embedding is also referred to as the graphing functor [14].

Remark 1.1. Another way of generalising the concept of relation between sets to "relations" between categories is via the notion of profunctor from $\mathbf{C}$ to $\mathbf{D}$ : it is an ordinary functor $\mathcal{F}: \mathbf{C} \times \mathbf{D}^{o p} \rightarrow$ Set (here Set could be replaced by another suitable monoidal category $\mathcal{V}$ ). Composition is via the left Kan extension along the Yoneda embedding [5]. The resulting structure is not a category but a bicategory. Multirelations from $C$ to $D$ can be seen as profunctors where $C$ and $D$ are considered as discrete categories. Formalising this observation yields a biequivalence from $\operatorname{Span}($ Set $)$ to the bicategory of profunctors between discrete categories [27]. This fact relies on a special property of Set, namely the equivalence of categories Set $\downarrow C \cong[C$, Set $]$, and therefore does not generalise readily.

Spans occur in very different contexts and often allow succinct characterisations of various concepts. For example: Katis, Sabadini and Walters [24] use spans to model systems with boundary (see also [15]); bisimulation has been captured as a span of open maps [23] as well as a span of coalgebra morphisms [2] an internal category is a monad in the bicategory of spans [4]; interaction categories [1] can be seen as examples of process categories [8], which are certain quotients of span bicategories; Mackey functors are coproduct preserving functors from the span-category [32].

\section{Colimits in the SPAn CATEgory}

We have seen that $\mathbf{C}$ embeds into $\operatorname{Sp}(\mathbf{C})$ and spans can be thought of as generalised relations. It is well-known (see, for example, [14, 1.911]) that in toposes colimits are preserved into the associated category of relations via the standard embedding. A natural question then is what conditions of $\mathbf{C}$ colimits are necessary and sufficient for them to be preserved by the 
embedding into $\operatorname{Sp}(\mathbf{C})$. We begin our investigation with the concrete case of coproducts. It turns out that this problem is closely related with the notion of extensive categories, where coproducts interact well with pullbacks.

The following, elementary definition of extensive categories makes this explicit [7].

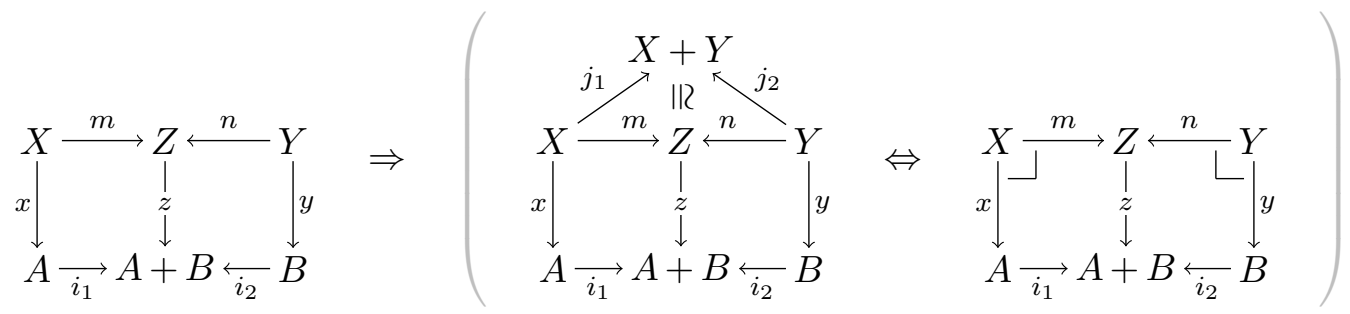

FiguRE 2. Extensivity condition

Definition 2.1. A category is extensive when

(1) it has finite coproducts;

(2) it has pullbacks along coproduct injections;

(3) given a diagram on the left of Figure 2 with the bottom row a coproduct diagram, the top row is a coproduct diagram if only if the two squares are pullbacks.

The relevant observation about coproducts in extensive categories is that the universal property of coproducts does not only apply to morphisms of the category itself but actually extends to spans. More precisely, given an ordinary coproduct $A-i_{1} \rightarrow A+B \leftarrow i_{2}-B$ and a pair of morphisms $f: A \rightarrow C$ and $g: B \rightarrow C$ there exists unique mediating morphism $[f, g]: A+B \rightarrow C$; given a coproduct in an extensive category and a pair of spans $A-(x, h) \rightarrow C$ and $B-(y, k) \rightarrow C$ there exists a unique mediating span $A+B-(x+y,[h, k]) \rightarrow C$. This was already noticed by Lindner [32].

More can be said: it turns out that if a coproduct in $\mathbf{C}$ is also a coproduct in $\operatorname{Sp}(\mathbf{C})$ then it satisfies the extensivity condition of Figure 2. Hence, the extensivity condition characterises the universal property of coproducts in the "larger universe" of spans. Summarizing, a coproduct in $\mathbf{C}$ satisfies the extensivity condition if and only if it is a coproduct in $\operatorname{Sp}(\mathbf{C})$.

Proposition 2.2. Let $\mathbf{C}$ be a category with coproducts and pullbacks. Then $\mathbf{C}$ is extensive if and only if the graphing functor $\Gamma: \mathbf{C} \rightarrow \mathrm{Sp}(\mathbf{C})$ preserves coproducts.

Proof. The fact that $\Gamma$ preserves coproducts when $\mathbf{C}$ is extensive was shown by Lindner 32 , Lemma 3].

For the converse, assume that $\Gamma: \mathbf{C} \rightarrow \mathrm{Sp}(\mathbf{C})$ preserves coproducts. First we shall show that coproducts in $\mathbf{C}$ are stable under pullback. Let $A-i_{A} \rightarrow A+B \leftarrow i_{B}-B$ be a coproduct diagram and $z: Z \rightarrow A+B$. Consider the diagram below.

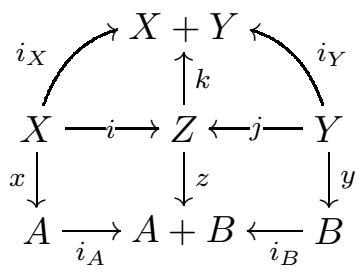


First assume that $A \leftarrow x-X-i \rightarrow Z$ and $Z \leftarrow j-Y-y \rightarrow B$ are pullbacks of $A-i_{A} \rightarrow A+B \leftarrow z-Z$ and $Z-z \rightarrow A+B \leftarrow i_{B}-B$, respectively. The existence of $k: Z \rightarrow X+Y$ with $k i=i_{X}$ and $k j=i_{Y}$ follows from the fact that $\left\langle\Gamma i_{A}, \Gamma i_{B}\right\rangle$ is a coproduct diagram in $\operatorname{Sp}(\mathbf{C})$. The universal property of $X+Y$ in $\mathbf{C}$ implies that $k[i, j]=\mathrm{id}_{X+Y}(*)$. The universal property of $A+B$ in $\operatorname{Sp}(\mathbf{C})$ implies that $(z, \mathrm{id})=(z,[i, j] k)$ in $\operatorname{Sp}(\mathbf{C})$, which implies the existence of an isomorphism $\varphi: Z \rightarrow Z$ with $[i, j] k=\varphi(* *)$. It now follows from $(*)$ that $k$ is split epi and from $(* *)$ that it is mono; thus $k$ is an isomorphism.

To verify the second part of the extensivity condition, consider the boundary of the diagram above. We need to show that $A \leftarrow x-X-i_{X} \rightarrow X+Y$ and $X+Y \leftarrow i_{Y}-Y-y \rightarrow B$ are pullbacks of $A-i_{A} \rightarrow A+B \leftarrow x+y-X+Y$ and $X+Y-x+y \rightarrow A+B \leftarrow i_{B}-B$. Now since $A+B$ is a coproduct in $\operatorname{Sp}(\mathbf{C})$ we get the existence of the interior part of the diagram, with the two squares pullbacks. By the argument in the previous paragraph $Z$ is the coproduct of $X$ and $Y$ and so $k$ is an isomorphism.

The main insight that can be gained by inspection of this proof is a correspondence between existence and uniqueness of mediating spans on the one hand and the two directions of the bi-implication of the Extensivity Condition in Figure 2 on the other hand. An analogous correspondence will recur later in the development of the main result in the bicategory of spans. The necessity of the bicategorical setting when considering arbitrary colimits is the topic of the remainder of this section.

2.1. The abstract span category is not enough. Proposition 2.2 could tempt one to try a generalisation to pushouts in the sense that a pushout is "well-behaved" in $\mathbf{C}$ if and only if it is preserved by $\Gamma$ as a pushout in $\operatorname{Sp}(\mathbf{C})$.

A good candidate for a "well-behaved" pushout is given by the notion of Van Kampen square, which appeared as part of the definition of adhesive categories [28]; indeed this definition was partly motivated by the extensivity condition of coproducts.

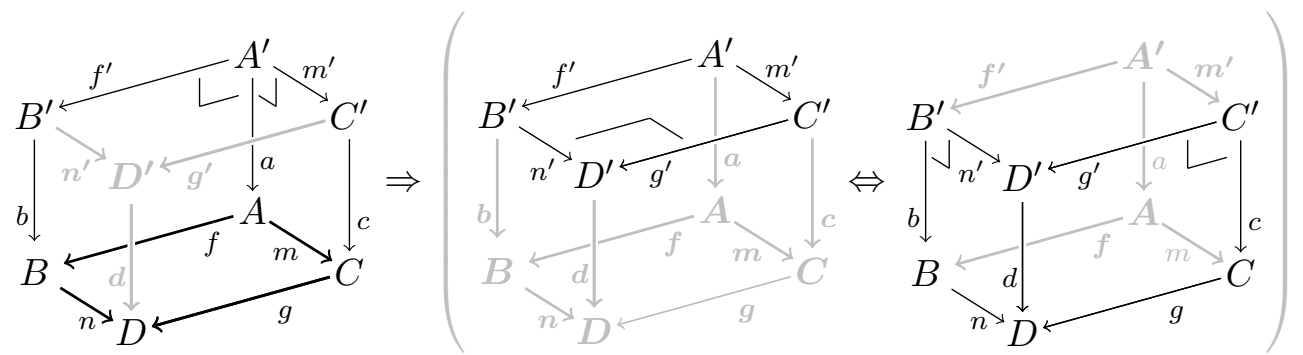

FiguRE 3. Van Kampen square

Definition 2.3 (Van Kampen square). A commutative square $B \leftarrow f-A-m \rightarrow C, B-n \rightarrow$ $D \leftarrow g-C$ is Van Kampen when for each commutative cube as illustrated in Figure 3 on the left that has pullback squares as rear faces, its top face is a pushout square if and only if its front faces are pullback squares (see Figure 3 ).

A category is adhesive when it has pushouts along monomorphisms and these are Van Kampen squares.

Differently from coproducts in extensive categories, Van Kampen squares do not induce pushouts via inclusion into the span category. Roughly, the reason for this is that for every 
pair of concrete $\mathbf{C}$-spans $C \leftarrow l-X-r \rightarrow D$ and $C \leftarrow l^{\prime}-X^{\prime}-r^{\prime} \rightarrow D$, there may be several different isomorphisms $\varphi_{1}, \varphi_{2}, \ldots: X \rightarrow X^{\prime}$ which witness that $(l, r): C \rightarrow D$ and $\left(l^{\prime}, r^{\prime}\right): A \rightarrow B$ are the same arrow in $\operatorname{Sp}(\mathbf{C})$.

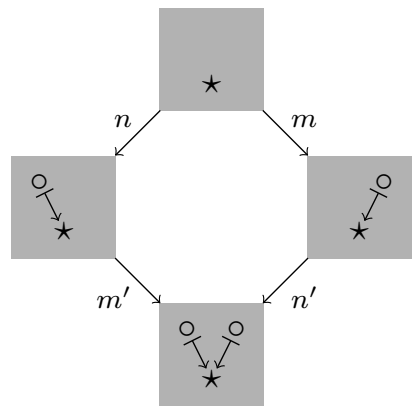

(A) pushout in $\mathbf{C}$

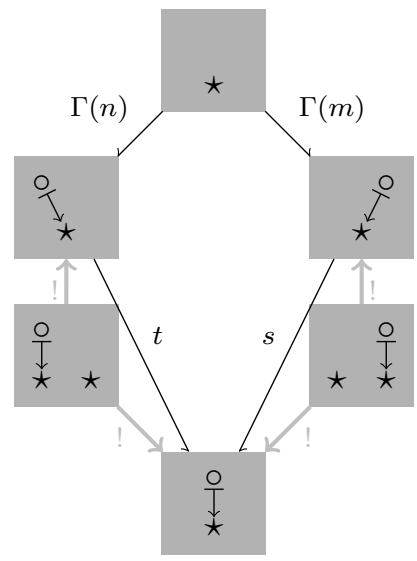

(B) cocone in $\operatorname{Sp}(\mathbf{C})$

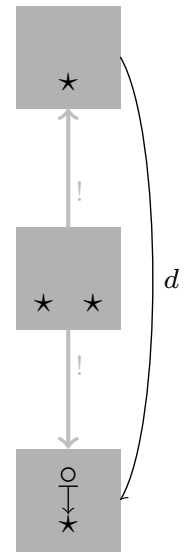

(C) diagonal

Figure 4. Van Kampen square in $\mathbf{C}=[\cdot \rightarrow \cdot$, Set $]$ and a cocone of spans

Our counterexample is a pushout in the category Set-arrows, i.e. the functor category $[\cdot \rightarrow \cdot$, Set], which is adhesive [29]. The objects of this category are functions. They will be depicted as gray boxes in which all input-output pairs are connected by arrows of the form ' $\mapsto$ ' (see Figure 4); each element of the domain is rendered as a small circle ' $\circ$ ', while each element of the codomain is represented by a star ' $\star$ '.

Now Figure 4](A) is a pushout of a pair of monomorphisms and hence a Van Kampen square. Consider the cocone for the "same" span of morphisms in $\mathrm{Sp}(\mathbf{C})$ described in Figure [4](B) in the latter figure the gray arrows belong to $\mathbf{C}$ while the arrows $s$ and $t$ belong to $\operatorname{Sp}(\mathbf{C})$. Figure $4(\mathrm{C})]$ shows the diagonal span $d$ of this square. Observe that any concrete representative of the diagonal span $d$ actually has a non-trivial symmetry group which consists of one non-identity automorphism as well as the identity.

As illustrated in Figure 5, each of the mediating spans can be constructed in the category $\mathbf{C}$ by means of the Van Kampen square property. The cocone from Figure 4](B) consisting of $s$ and $t$ is now pointing to the top. Commutativity of the cocone in $\operatorname{Sp}(\mathbf{C})$ gives a pair of $\mathbf{C}$-pullbacks in the back of each of the diagrams in Figure 5. Depending on choices of pullbacks and witnesses for the abstract equality of the composite spans, we obtain two different diagrams over which we can take a pushout. (In Figure 5, we tried to express this fact by "switching" of the two stars in the carrier of right span of the cocone.) These two diagrams on top of a cube yield two different mediating arrows in the category of spans by taking the pushout in $\mathbf{C}$. In the end, we obtain two different mediating spans to the same cocone in $\operatorname{Sp}(\mathbf{C})$, and thus $\cdot-\Gamma\left(n^{\prime}\right)-\cdot\left\llcorner\Gamma\left(m^{\prime}\right)-\cdot\right.$ cannot be a pushout of . $\angle \Gamma(m)-\cdot-\Gamma(n) \rightarrow \cdot$

Remark 2.4. To solve the problem of non-trivial symmetry groups of spans one could try to restrict to partial map spans, i.e. those spans with a monomorphisms from the carrier into the domain. This however would yield a (properly) weaker notion, which one could call partial Van Kampen square [16. In fact, in the category of sets and, more generally, any 

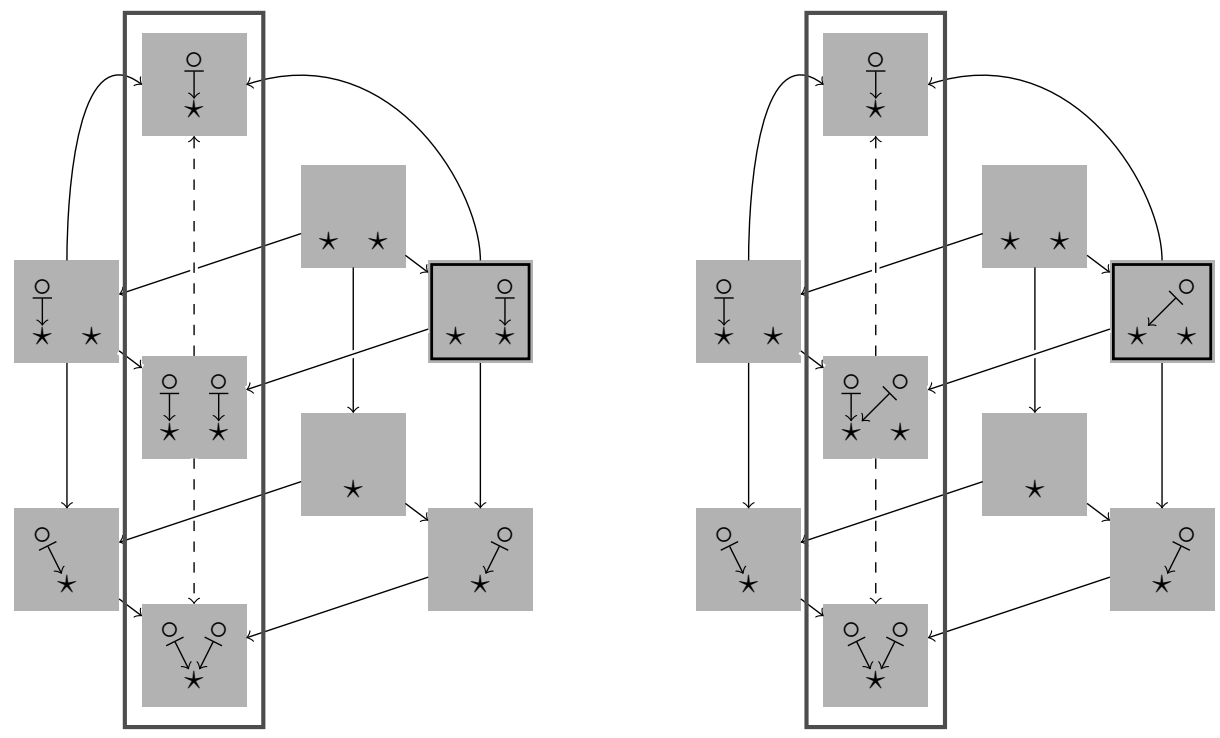

Figure 5. Two different mediating spans to the same cocone of spans

elementary topos, all colimits are partial Van Kampen colimits while there are examples of pushouts in Set, which are not Van Kampen [29].

The only canonical way to "tame" the non-trivial symmetry groups of spans is to keep track of the involved isomorphisms, i.e. we have to work in a bicategory $\operatorname{Span}(\mathbf{C})$ of (concrete) spans in $\mathbf{C}$.

It turns out that by moving to the setting of bicategories (recalled in the proceeding section) we obtain not only that Van Kampen squares characterise those pushouts that are preserved by $\Gamma$ but that a general Van Kampen condition (introduced in Section 4) characterises those colimits that satisfy the bicategorical universal property of colimits.

\section{Bichtegories}

Here we introduce background on bicategories [4, 5, 27] and some notational conventions. Our focus is the bicategory of spans over a category $\mathbf{C}$ with a choice of pullbacks (see Example 3.3). This allows us to avoid unnecessary book-keeping by considering only those bicategories that satisfy the identity axioms strictly. It is only a cosmetic choice, the development can be easily adapted to the standard setting of bicategories.

Definition 3.1 (Strictly unitary bicategories). A strictly unitary (su) bicategory $\mathscr{B}$ consists of:

- a collection ob $\mathscr{B}$ of objects;

- for $A, B \in$ ob $\mathscr{B}$ a category $\mathscr{B}(A, B)$, the objects and arrows of which are called, respectively, the arrows and the 2 -cells of $\mathscr{B}$. Composition is denoted by $\odot$ and referred to as vertical composition. Given $(f: A \rightarrow B) \in \mathscr{B}(A, B)$, its identity 2-cell will be denoted $\iota_{f}: f \rightarrow f$. Each $\mathscr{B}(A, A)$ contains a special object $\operatorname{id}_{A}: A \rightarrow A$, called the identity arrow; 
- for $A, B, C \in$ ob $\mathscr{B}$, a functor $c_{A, B, C}: \mathscr{B}(A, B) \times \mathscr{B}(B, C) \rightarrow \mathscr{B}(A, C)$ called horizontal composition. On objects, $c_{A, B, C}\langle f, g\rangle$ is written $g \circ f$, while on arrows $c_{A, B, C}\langle\gamma, \delta\rangle$ it is $\delta * \gamma$. For any $f: A \rightarrow B$ we have $\operatorname{id}_{B} \circ f=f=f \circ \operatorname{id}_{A}$;

- for $A, B, C, D \in$ ob $\mathscr{B}$, arrows $f: A \rightarrow B, g: B \rightarrow C$ and $h: C \rightarrow D$ an associativity natural isomorphism $\alpha_{A, B, C, D}(f, g, h): h \circ(g \circ f) \rightarrow(h \circ g) \circ f$. It satisfies the coherence axioms: for any composable $f, g, h, k$, we have $\alpha_{f, \text { id }, g}=\iota_{g \circ f}$ and also that the following 2-cells are equal:

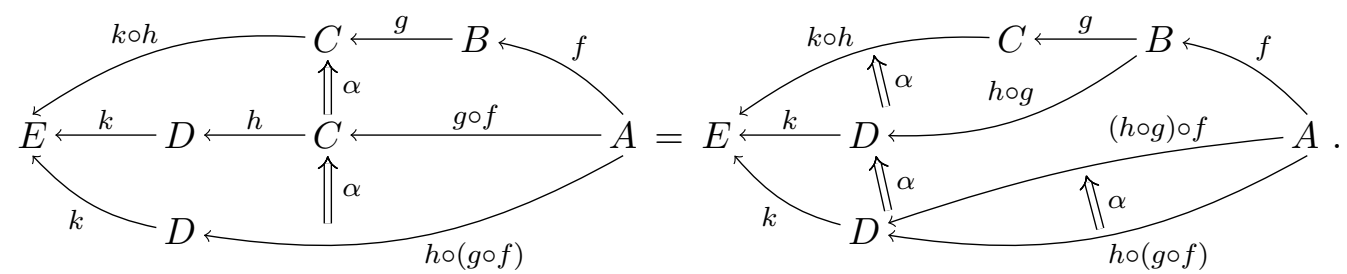

Example 3.2. Any (ordinary) category $\mathbf{C}$ is a (SU-)bicategory with trivial 2-cells.

As we have seen, composition in the category $\operatorname{Sp}(\mathbf{C})$ is obtained via pullback. Because of the universal property of pullbacks and the fact that the arrows of $\operatorname{Sp}(\mathbf{C})$ are abstract spans, composition defined in this fashion is well-defined. Instead, in order to compose concrete spans we shall need to assume some choice of pullback in $\mathbf{C}$; this means that for any cospan $X-f \rightarrow Z \leftarrow g-Y$ there is a chosen object $X \times{ }_{Z} Y$ and span $X \leftarrow \pi_{1}-X \times{ }_{Z} Y-\pi_{2} \rightarrow Y$ that together with $f$ and $g$ forms a pullback square. Moreover we assume that the choice preserves identities: if $f$ is $\operatorname{id}_{X}$ then $X \times_{Z} Y=Y$ and $\pi_{1}=\operatorname{id}_{Y}$, and analogously for $g$. This is a completely harmless assumption since the identity of the chosen pullback diagram for any cospan is insignificant: any two choices are equivalent.

Example 3.3 (Span bicategory [4]). Assume that $\mathbf{C}$ has a choice of pullbacks that preserves identities. $\operatorname{Span}(\mathbf{C})$ has:

- as objects, the objects of $\mathbf{C}$, i.e. ob $\operatorname{Span}(\mathbf{C})=$ ob $\mathbf{C}$;

- as arrows from $C$ to $D$, the $\mathbf{C}$-spans $C \leftarrow l-X-r \rightarrow D$. The composition with another span $D \leftarrow l^{\prime}-Y-r^{\prime} \rightarrow E$ is obtained via the chosen pullback as illustrated below; however this composition is only associative up to canonical isomorphism. The identity on an object $C$ is the span $C \leftarrow \mathrm{id}-C-\mathrm{id} \rightarrow C$.

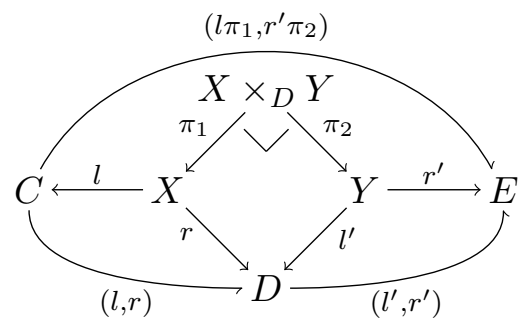

- its 2-cells $\xi:(l, r) \rightarrow\left(l^{\prime}, r^{\prime}\right)$ are $\mathbf{C}$-arrows $\xi: X \rightarrow X^{\prime}$ between the respective carriers such that $l^{\prime} \circ \xi=l$ and $r^{\prime} \circ \xi=r$.

For our purposes it suffices to consider strict homomorphisms between SU-bicategories.

Definition 3.4 (Strict homomorphisms [4]). Let $\mathscr{A}$ and $\mathscr{B}$ be su-bicategories. A strict homomorphism $\mathcal{F}: \mathscr{A} \rightarrow \mathscr{B}$ consists of a function $\mathcal{F}:$ ob $\mathscr{A} \rightarrow$ ob $\mathscr{B}$ and a family of functors $\mathcal{F}(A, B): \mathscr{A}(A, B) \rightarrow \mathscr{B}(\mathcal{F} A, \mathcal{F} B)$ such that: 
(i) for all $A \in \mathscr{A}, \mathcal{F}\left(\operatorname{id}_{A}\right)=\operatorname{id}_{\mathcal{F} A}$;

(ii) for all $f: A \rightarrow B, g: B \rightarrow C$ in $\mathscr{A}, \mathcal{F}(g \circ f)=\mathcal{F}(g) \circ \mathcal{F}(f)$;

(iii) $\mathcal{F} \alpha_{A, B, C, D}=\alpha_{\mathcal{F} A, \mathcal{F} B, \mathcal{F} C, \mathcal{F} D}$.

Example 3.5. The following strict homomorphisms will be of interest to us:

- the covariant embedding $\Gamma: \mathbf{C} \rightarrow \operatorname{Span}(\mathbf{C})$ which acts as the identity on objects and takes an arrow $f: C \rightarrow D$ to its graph (id, $f$ ): $C \rightarrow D$. It allows to consider the objects and morphisms in the "universe" $\mathbf{C}$ in the "larger universe" $\operatorname{Span}(\mathbf{C})$;

- $\Gamma \mathcal{F}: \mathbf{J} \rightarrow \operatorname{Span}(\mathbf{C})$ where $\mathcal{F}: \mathbf{J} \rightarrow \mathbf{C}$ is a functor. It allows to lift every diagram in $\mathbf{C}$ to a diagram in $\operatorname{Span}(\mathbf{C})$;

- given a su-bicategory $\mathscr{B}$ and $B \in \mathrm{ob} \mathscr{B}$, we shall abuse notation and denote the strict homomorphism from $\mathbf{J}$ to $\mathscr{B}$ which is constant at $B$ by $\Delta B$. It is used to define conical bi-colimits for diagrams. Note that in the case of $\mathscr{B}=\operatorname{Span}(\mathbf{C}), " \Delta=\Gamma \Delta "$.

Definition 3.6 (Lax transformations). Given strict homomorphisms $\mathcal{F}, \mathcal{G}: \mathscr{A} \rightarrow \mathscr{B}$ between su-bicategories, a (lax) transformation consists of arrows $\kappa_{A}: \mathcal{F} A \rightarrow \mathcal{G A}$ for all $A \in \mathscr{A}$ and 2-cells $\kappa_{f}: \mathcal{G} f \circ \kappa_{A} \Rightarrow \kappa_{B} \circ \mathcal{F} f$ for all $f: A \rightarrow B$ in $\mathscr{A}$ (illustrated below)

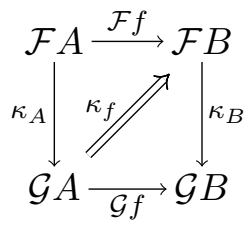

such that:

(i) $\kappa_{\mathrm{id}_{A}}=\iota_{\kappa_{A}}$ for each $A \in \mathscr{A}$;

(ii) for any $f: A \rightarrow B, g: B \rightarrow C$ in $\mathscr{A}$, the following 2-cells are equal:

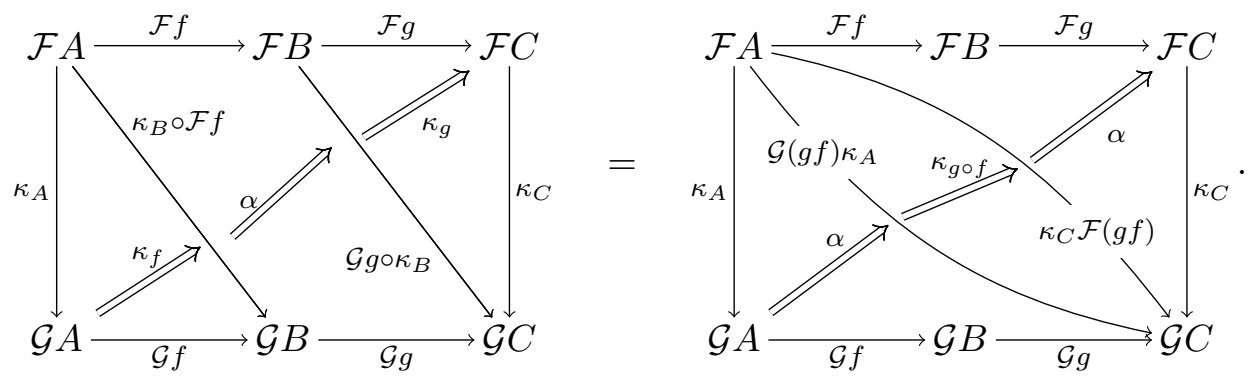

A transformation is said to be strong when all the $\kappa_{f}$ are invertible 2-cells. Given $B \in \mathscr{B}$ and a homomorphism $\mathcal{M}: \mathbf{J} \rightarrow \mathscr{B}$, a pseudo-cocone $\lambda: \mathcal{M} \rightarrow \Delta B$ is a synonym for a strong transformation $\lambda: \mathcal{M} \rightarrow \Delta B$.

Because bicategories have 2-cells, there are morphisms between transformations. They are called modifications and are defined as follows.

Definition 3.7 (Modifications [4, 26]). Given natural transformations $\kappa, \lambda$ from $\mathcal{F}$ to $\mathcal{G}$, a modification $\Xi: \kappa \rightarrow \lambda$ consists of 2-cells $\Xi_{A}: \kappa_{A} \rightarrow \lambda_{A}$ for $A \in \mathscr{A}$ such that, for all 


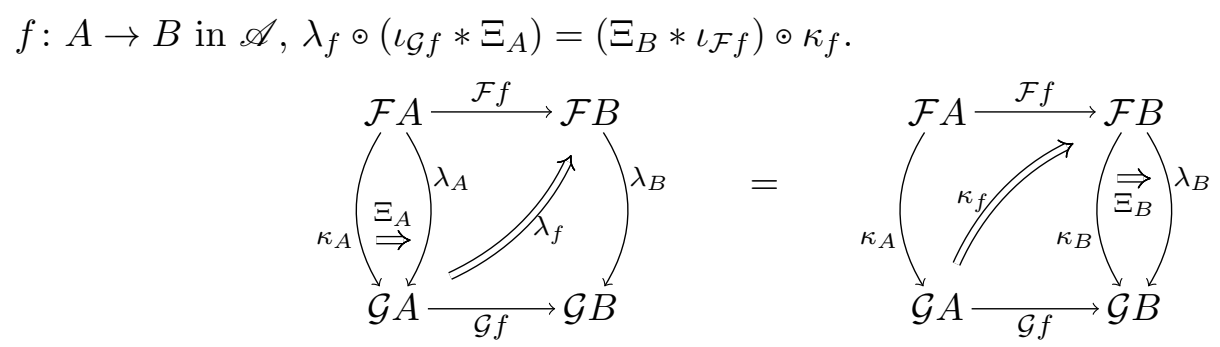

Composition is componentwise, the identity modification on $\kappa$ is $I_{\kappa}=\left\{\iota_{\kappa_{A}}\right\}_{A \in \mathscr{A}}$.

Given su-bicategories $\mathscr{A}$ and $\mathscr{B}$, let $\operatorname{Hom}_{l}[\mathscr{A}, \mathscr{B}]$ denote the su-bicategory of homomorphisms, lax transformations and modifications. Let Hom $[\mathscr{A}, \mathscr{B}]$ denote the corresponding su-bicategory with arrows the strong transformations.

\section{VAN KAMPEN COCONES}

The extensivity condition for coproducts and the notion of Van Kampen condition for pushouts are both examples (for particular colimits) of a more general condition. Colimit diagrams that satisfy it are called Van Kampen cocones. Here we give this definition together with an elementary characterisation.

Let us consider coproducts as a motivating example. A coproduct diagram

$$
A-i_{1} \rightarrow A+B \leftarrow i_{2}-B
$$

in a category $\mathbf{C}$ is a cocone of the two-object diagram $\langle A, B\rangle$. If $\mathbf{C}$ has chosen pullbacks (along coproduct injections) then $i_{1}$ yields a functor $i_{1}^{*}: \mathbf{C} \downarrow(A+B) \rightarrow \mathbf{C} \downarrow A$ that takes an arrow $x: X \rightarrow A+B$ to its pullback $i_{1}{ }^{*} x: i_{1}{ }^{*} X \rightarrow A$ along $i_{1}$-similarly for $i_{2}$. Then $x \mapsto\left\langle i_{1}{ }^{*} x, i_{2}{ }^{*} x\right\rangle$ defines the functor $\left\langle i_{1}{ }^{*}{ }, i_{2}{ }^{*}{ }^{*}\right\rangle:(\mathbf{C} \downarrow A+B) \rightarrow(\mathbf{C} \downarrow A \times \mathbf{C} \downarrow B)$ on objects. The coproduct $A+B$ satisfies the extensivity condition exactly when this functor is an equivalence of categories (see [7]).

The situation readily generalises as follows: replace $\left\langle i_{1}, i_{2}\right\rangle$ by any cocone $\kappa: \mathcal{D} \rightarrow \Delta A$ from a functor $\mathcal{D}: \mathbf{J} \rightarrow \mathbf{C}$ to an object $A$ in a category $\mathbf{C}$ with (enough) pullbacks. Any arrow $x: X \rightarrow A$ induces a natural transformation $\Delta x: \Delta X \rightarrow \Delta A$ in the obvious way. Since also $\kappa: \mathcal{D} \rightarrow \Delta A$ is a natural transformation, $\Delta x$ can be pulled back along $\kappa$ in the functor category $[\mathbf{J}, \mathbf{C}]$ yielding a natural transformation $\kappa^{*}(\Delta x): \kappa^{*}(\Delta X) \rightarrow \mathcal{D}$.

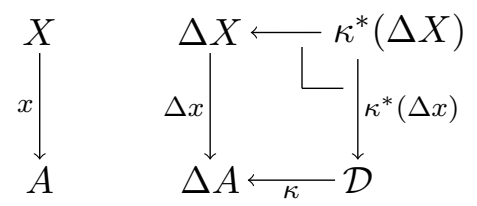

The described operation extends to a functor $\kappa^{*}\left(\Delta_{\sqcup}\right)$ from $\mathbf{C} \downarrow A$ to (a full subcategory of) $[\mathbf{J}, \mathbf{C}] \downarrow \mathcal{D}$ using the universal property of pullbacks; it takes morphisms with codomain $A$ to cartesian transformations with codomain $\mathcal{D}$.

Definition 4.1 (Cartesian transformations). Let $\mathcal{E}, \mathcal{D} \in[\mathbf{J}, \mathbf{C}]$ be functors and let $\tau: \mathcal{E} \rightarrow \mathcal{D}$ be a natural transformation. Then $\tau$ is cartesian when all the naturality squares are pullback 
squares, i.e. if the pair $\mathcal{E}_{i} \leftarrow \tau_{i}-\mathcal{D}_{i}-\mathcal{D}_{u} \rightarrow \mathcal{D}_{j}$ is a pullback of $\mathcal{E}_{i}-\mathcal{E}_{u} \rightarrow \mathcal{E}_{j} \leftarrow \tau_{j}-\mathcal{D}_{j}$ for all $u: i \rightarrow j$ in $\mathbf{J}$.

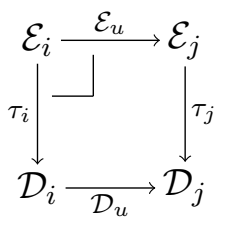

Let $[\mathbf{J}, \mathbf{C}] \downarrow \mathcal{D}$ be the slice category over $\mathcal{D}$, which has natural transformations with codomain $\mathcal{D}$ as objects. Let $[\mathbf{J}, \mathbf{C}] \Downarrow \mathcal{D}$ denote the full subcategory of $[\mathbf{J}, \mathbf{C}] \downarrow \mathcal{D}$ with the cartesian transformations as objects.

Definition 4.2 (Van Kampen cocones). Let $\mathcal{D}: \mathbf{J} \rightarrow \mathbf{C}$ be a functor, and let $\kappa: \mathcal{D} \rightarrow \Delta A$ be a cocone such that pullbacks along each $\kappa_{i}$ exist $(i \in \mathbf{J})$. Then $\kappa$ is Van Kampen (VK) if the functor $\kappa^{*}\left(\Delta_{\bullet}\right): \mathbf{C} \downarrow A \rightarrow[\mathbf{J}, \mathbf{C}] \Downarrow \mathcal{D}$ is an equivalence of categories.

Extensive and adhesive categories have elementary characterisations that are special cases of the following.

Proposition 4.3 (Elementary VK characterisation). Suppose that $\mathbf{C}$ has pullbacks and $\mathbf{J}$ colimits, $\mathcal{D}: \mathbf{J} \rightarrow \mathbf{C}$ is a functor and $\kappa: \mathcal{D} \rightarrow \Delta A$ a cocone such that $\mathbf{C}$ has pullbacks along $\kappa_{i}(i \in \mathbf{J})$. Then $\kappa: \mathcal{D} \rightarrow \Delta A$ is Van Kampen iff for every cartesian transformation $\tau: \mathcal{E} \rightarrow \mathcal{D}$, arrow $x: X \rightarrow A$ and cocone $\beta: \mathcal{E} \rightarrow \Delta X$ such that $\kappa \circ \tau=\Delta x \circ \beta$, the following are equivalent:

(i) $\beta: \mathcal{E} \rightarrow \Delta X$ is a $\mathbf{C}$-colimit;

(ii) $\mathcal{D}_{i} \leftarrow \tau_{i}-\mathcal{E}_{i}-\beta_{i} \rightarrow X$ is a pullback of $\mathcal{D}_{i}-\kappa_{i} \rightarrow A \leftarrow x-X$ for all $i \in \mathbf{J}$.

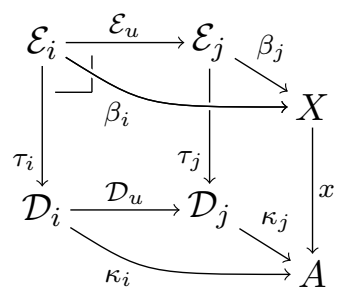

Proof. The proof is a straightforward generalisation of the corresponding characterisation of Van Kampen squares [29, Proposition 2.6]. Assume (i) $\Leftrightarrow($ ii) as well as existence of $\mathbf{J}$ colimits. Essential surjectivity of $\kappa^{*}$ follows easily from (i) $\Rightarrow$ (ii). The fact that $\kappa^{*}$ is faithful follows from (ii) $\Rightarrow$ (i) and it is full because of existence of $\mathbf{J}$-colimits.

Conversely, in the presence of $\mathbf{J}$-colimits, $\kappa^{*}$ has a left adjoint defined in the obvious way by taking the appropriate colimits. Then if $\kappa^{*}$ is an equivalence, it follows that the unit and counit of this adjunction are isomorphisms. Now (i) $\Rightarrow$ (ii) is implied by the fact that the unit is an isomorphism and (ii) $\Rightarrow(\mathrm{i})$ is a consequence of the counit being an isomorphism.

Cockett and Guo's 9] definition of Van Kampen colimits is the equivalence of (i) and (ii) in our Proposition 4.3. If the relevant pullbacks and colimits exist then clearly the two definitions are equivalent.

Remark 4.4. With the assumptions of Proposition 4.3, any Van Kampen cocone $\kappa: \mathcal{D} \rightarrow$ $\Delta A$ is a colimit diagram of $\mathcal{D}$ in $\mathbf{C}\left(\right.$ take $\tau=\operatorname{id}_{\mathcal{D}}$ and $\left.x=\mathrm{id}_{A}\right)$.

Example 4.5. The following well-known concepts are examples of vK-cocones:

(i) a strict initial object is a VK-cocone for the functor from the empty category; 
(ii) a coproduct diagram in an extensive category [7] is a VK-cocone for a functor from the discrete two object category;

(iii) a regular epimorphism $p: E \rightarrow B$ in a regular category is the Van Kampen cocone of its kernel pain $r, s: P \rightrightarrows E$, i.e. in any commuting diagram of the following form

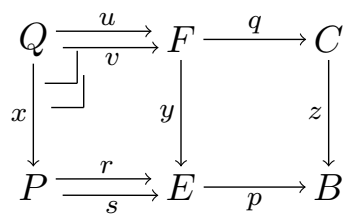

in which $u, v: Q \rightrightarrows F$ is a kernel pair (of some morphism) and the squares on the left are pullbacks, the morphism $q$ is the coequaliser of $u, v: Q \rightrightarrows F$ if and only if the right square is a pullback. This is a direct consequence of a result of Barr and Kock [3, Example 6.10]. Notice that in this case the colimits in question are coequalisers of kernel pairs; this means that we implicitly restrict the category on the right hand side of the equivalence in Definition 4.2 to the full subcategory with objects the kernel pairs.

A VK-cocone from a span is a Van Kampen square (see Definition 2.3). In the left hand diagram in Figure 3, the two arrows $B \leftarrow f-A-m \rightarrow C$ describe a diagram from the three object category $\cdot \leftarrow \cdot \rightarrow \cdot$, and the cospan $B-n \rightarrow D \leftarrow g-C$ gives a cocone for this diagram. That the back faces are pullback squares means that we have a cartesian transformation from $B^{\prime} \leftarrow f^{\prime}-A^{\prime}-m^{\prime} \rightarrow C$ to $B \leftarrow f-A-m \rightarrow C$. Adhesive categories are thus precisely categories with pullbacks in which pushouts along monomorphisms exist and are vK-cocones.

\section{Colimits in the SPAN BiCATEgory}

In Section 2 we showed that coproducts that satisfy the extensivity condition are precisely those that are preserved into the span category (Proposition 2.2) when the latter is welldefined, i.e. if all pullbacks exist. This correspondence failed when we considered pushouts which are Van Kampen squares - a condition for pushouts analogous to the extensivity condition for coproducts. The main result of this paper, Theorem 6.3, asserts that the correspondence holds not only for pushouts but for general Van Kampen cocones when considering the canonical embedding into the span bicategory providing that it exists, i.e. the ambient category has all pullbacks. In order to show this we shall need to recall the canonical notion of colimit in bicategories in general and in bicategories of spans in particular.

To give the flavour of the correspondence between VK-cocones in $\mathbf{C}$ and colimits in $\operatorname{Span}(\mathbf{C})$ we outline how Van Kampen squares induce (bi)pushout squares via the embedding $\Gamma$. An illustration of this is given in Figure 6 ,

At the base of Figure G(A) is (the image of) a $\mathbf{C}$-span $B \leftarrow f-A-m \rightarrow C$ in $\operatorname{Span}(\mathbf{C})$. Further, if $\left(b, b^{\prime}\right): B \rightarrow E$ and $\left(c, c^{\prime}\right): C \rightarrow E$ are a pseudo-cocone for $B \leftarrow \Gamma f-A-\Gamma m \rightarrow C$ in Span $(\mathbf{C})$ then taking pullbacks of $b$ along $f$ and $c$ along $m$ (in $\mathbf{C}$ ) yields isomorphic objects over $A$, say $a_{b}$ and $a_{c}$; as a result we obtain two pullback squares that will be the back faces of a commutative cube.

\footnotetext{
${ }^{1}$ The kernel pair of a morphism is obtained by pulling it back along itself.
} 


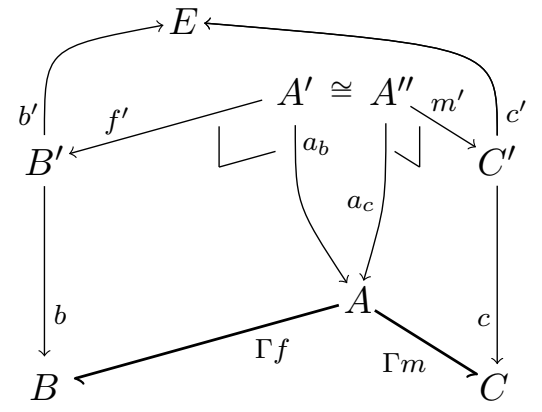

(A) $\operatorname{Span}(\mathbf{C})$-cocone of the $\mathbf{C}$ span $B \leftarrow f-A-m \rightarrow C$

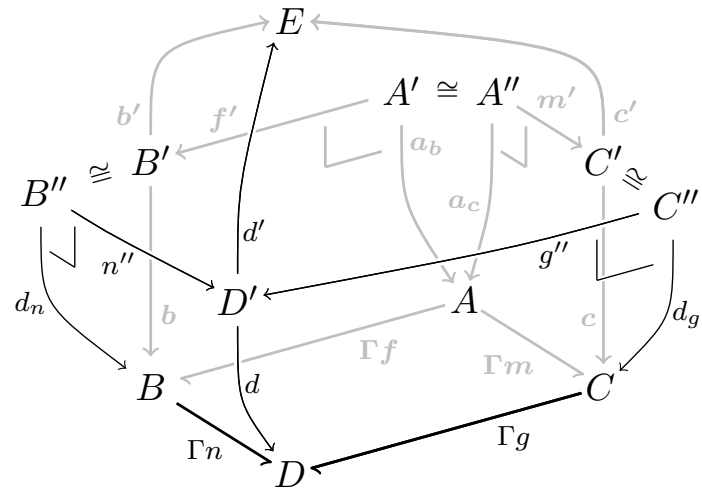

(B) Mediating $\operatorname{Span}(\mathbf{C})$-morphism from the C-cocone $B-n \rightarrow D \leftarrow g-C$

FiguRE 6. Cocones and mediating morphisms consisting of spans

Next, let the bottom of Figure 6 (B) be (the image of) a commuting $\mathbf{C}$-square, thus yielding another pseudo-cocone of $B<\Gamma f-A-\Gamma m \rightarrow C$, namely $B-\Gamma n \rightarrow D<\Gamma g-C$. If there is a mediating morphism $\left(d, d^{\prime}\right): D \rightarrow E$ to $B-\left(b, b^{\prime}\right) \rightarrow E\left\llcorner\left(c, c^{\prime}\right)-E\right.$ of Figure 6 (A) then pulling back $d$ along $n$ and $g$ results in morphisms $d_{n}$ and $d_{g}$, which are isomorphic to $b$ and $c$; the resulting pullback squares provide the front faces of a cube.

Now, if $B-n \rightarrow D \leftarrow g-C$ is a vK-cocone of $B \leftarrow f-A-m \rightarrow C$ then such a mediating morphism can be constructed by taking $D^{\prime}$ as the pushout of $B^{\prime}$ and $C^{\prime}$ over either one of $A^{\prime}$ or $A^{\prime \prime}$. The morphisms $d: D^{\prime} \rightarrow E$ and $d^{\prime}: D^{\prime} \rightarrow D$ arise from the universal property of pushouts, everything commutes and the front faces are pullback squares because of the VKproperty. Further this mediating morphism is essentially unique, which means that given any other $\operatorname{span}\left(e, e^{\prime}\right): D \rightarrow E$ such that both $\left(b, b^{\prime}\right) \cong\left(e, e^{\prime}\right) \circ \Gamma n$ and $\left(c, c^{\prime}\right) \cong\left(e, e^{\prime}\right) \circ \Gamma g$ hold, the two spans $\left(e, e^{\prime}\right)$ and $\left(d, d^{\prime}\right)$ are isomorphic via a unique isomorphism.

Though this sketch lacks relevant technical details, it gives a good indication of the diagrams that are involved in the proof of the fact that Van Kampen squares in $\mathbf{C}$ induce bipushouts in $\operatorname{Span}(\mathbf{C})$. Moreover, also the converse holds, i.e. if the image of a pushout is a bipushout in $\operatorname{Span}(\mathbf{C})$ then it is a Van Kampen square.

5.1. Span bicolimits. Clearly any diagram in $\operatorname{Span}(\mathbf{C})$ can be "decomposed" into a diagram in $\mathbf{C}$ : each arrow in $\operatorname{Span}(\mathbf{C})$ gives two $\mathbf{C}$-arrows from a carrier object; moreover a 2-cell is a compatible $\mathbf{C}$-arrow between the carriers.

We shall start with further observations along these lines. Roughly we are able to "drop a dimension" in the following sense. First, it is easy to see that $[\mathbf{J}, \mathbf{C}]$ inherits a choice of pullbacks from C. In particular, it follows that $\operatorname{Span}([\mathbf{J}, \mathbf{C}])$ is a SU-bicategory. Now, given $\mathcal{F}, \mathcal{G} \in[\mathbf{J}, \mathbf{C}]$ we note that:

- spans of natural transformations from $\mathcal{F}$ to $\mathcal{G}$ correspond to lax transformations from $\Gamma \mathcal{F}$ to $\Gamma \mathcal{G}$; and

- morphisms of such spans are the counterpart of modifications.

The following lemma makes this precise. 
Lemma 5.1. There is a strict homomorphism

$$
\boldsymbol{\Gamma}: \operatorname{Span}([\mathbf{J}, \mathbf{C}]) \rightarrow \operatorname{Hom}_{l}[\mathbf{J}, \operatorname{Span}(\mathbf{C})]
$$

that takes $\mathcal{F} \in[\mathbf{J}, \mathbf{C}]$ to $\Gamma \mathcal{F}$ and is full and faithful on both arrows and 2-cells.

Proof. A span of natural transformations $(\varphi, \psi): \mathcal{F} \rightarrow \mathcal{G}$ with carrier $\mathcal{H}$ is mapped to a lax transformation from $\Gamma \mathcal{F}$ to $\Gamma \mathcal{G}$ as follows: for each $i \in \mathbf{J}$, we put $\kappa_{i}:=\left(\varphi_{i}, \psi_{i}\right): \mathcal{F}_{i} \rightarrow \mathcal{G}_{i}$, and for each morphism $u: i \rightarrow j$ in $\mathbf{J}$, we define a 2-cell $\kappa_{u}: \Gamma \mathcal{G}_{u} \circ \kappa_{i} \rightarrow \kappa_{j} \circ \Gamma \mathcal{F}_{u}$ as sketched below. More explicitly, by naturality of $\varphi$ we have that $\mathcal{F}_{u} \circ \varphi_{i}=\varphi_{j} \circ \mathcal{H}_{u}$ and so the arrow $\kappa_{u}: \mathcal{H}_{i} \rightarrow \mathcal{F}_{i} \times \mathcal{F}_{j} \mathcal{H}_{j}$ is the unique one satisfying $\varphi_{i}=\pi_{1} \circ \kappa_{u}$ and $\mathcal{H}_{u}=\pi_{2} \circ \kappa_{u}$. To check that $\kappa_{u}$ is a 2-cell it remains verify that $\psi_{j} \circ \pi_{2} \circ \kappa_{u}=\psi_{j} \circ \mathcal{H}_{u}=\mathcal{G}_{u} \circ \psi_{i}$, which follows by the naturality of $\psi$.

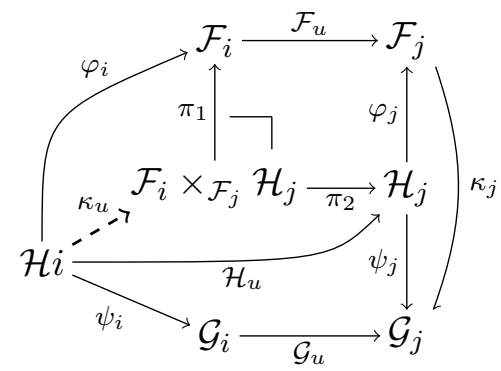

Further, a 2-cell between spans $(\varphi, \psi),\left(\varphi^{\prime}, \psi^{\prime}\right): \mathcal{F} \rightarrow \mathcal{G}$ with respective carriers $\mathcal{H}, \mathcal{H}^{\prime}$ is a natural transformation $\xi: \mathcal{H} \rightarrow \mathcal{H}^{\prime}$ satisfying both $\varphi^{\prime} \odot \xi=\varphi$ and $\psi^{\prime} \odot \xi=\psi$. This induces a modification $\left\{\xi_{i}\right\}_{i \in \mathbf{J}}: \boldsymbol{\Gamma}(\varphi, \psi) \rightarrow \boldsymbol{\Gamma}\left(\varphi^{\prime}, \psi^{\prime}\right)$.

It follows from the definition that $\kappa_{\mathrm{id}_{i}}=\iota_{\mathcal{H}_{i}}$. To check the second requirement of lax transformations (see Definition 3.6), consider two arrows $u: i \rightarrow j$ and $v: j \rightarrow k$ in J. Since $\alpha_{\mathcal{F}_{u}, \kappa_{j}, \mathcal{G} v}$ and $\alpha_{\kappa_{i}, \mathcal{G}_{u}, \mathcal{G} v}$ are identities, one merely has to show $\alpha \odot \kappa_{v \circ u}=\kappa_{v} * \iota_{\mathcal{F}_{u}} \odot \iota_{\mathcal{G} v} * \kappa_{u}$. The latter equation amounts to commutativity of the diagram below - a consequence of functoriality of $\mathcal{H}$.

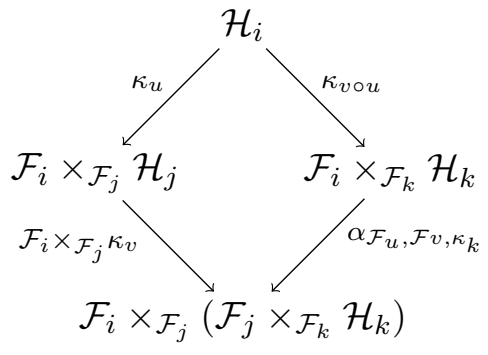

Faithfulness on arrows is immediate. Conversely, given a lax transformation $\kappa: \Gamma \mathcal{F} \rightarrow \Gamma \mathcal{G}$, we construct a functor $\mathcal{H}$ and natural transformations $\varphi: \mathcal{H} \rightarrow \mathcal{F}, \psi: \mathcal{H} \rightarrow \mathcal{G}$ such that $\boldsymbol{\Gamma}(\varphi, \psi)=\kappa$ as follows: let $\mathcal{H}_{i}$ be the carrier of the span $\kappa_{i}$ and $\mathcal{H}_{u}:=\pi_{2} \circ \kappa_{u}$, and further $\varphi_{i}$ and $\psi_{i}$ are the left and right component of span $\kappa_{i}$, respectively. Functoriality follows directly from the commutativity of the diagram above; naturality of $\varphi$ and $\psi$ follows from the fact that each $\kappa_{u}$ is a 2-cell.

Finally, a 2-cell between spans $(\varphi, \psi),\left(\varphi^{\prime}, \psi^{\prime}\right): \mathcal{F} \rightarrow \mathcal{G}$ with respective carriers $\mathcal{H}, \mathcal{H}^{\prime}$, is a natural transformation $\xi: \mathcal{H} \rightarrow \mathcal{H}^{\prime}$ satisfying both $\varphi^{\prime} \odot \xi=\varphi$ and $\psi^{\prime} \odot \xi=\psi$. To prove that such a 2-cell induces a modification $\left\{\xi_{i}\right\}_{i \in \mathbf{J}}: \boldsymbol{\Gamma}(\varphi, \psi) \rightarrow \boldsymbol{\Gamma}\left(\varphi^{\prime}, \psi^{\prime}\right)$ one needs to verify the equality $\kappa_{u}^{\prime} \odot \xi_{i}=\xi_{j} \odot \kappa_{u}$; this amounts to the commutativity of the diagram below. To see why the diagram commutes, consider the two projections $\mathcal{F}_{i} \leftarrow \pi_{1}-\mathcal{F}_{i} \times_{\mathcal{F}_{j}} \mathcal{H}_{j}^{\prime}-\pi_{2} \rightarrow \mathcal{H}_{j}^{\prime}$ : 
now one has $\pi_{1} \circ \kappa_{u}^{\prime} \circ \xi_{i}=\varphi_{i}=\pi_{1} \circ\left(\mathcal{F}_{i} \times_{\mathcal{F}_{j}} \xi_{j}\right) \circ \kappa_{u}$ because of $\varphi=\varphi^{\prime} \circ \xi$ and $\pi_{2} \circ \kappa_{u}^{\prime} \circ \xi_{i}=$ $\mathcal{H}_{u}^{\prime} \circ \xi_{i}=\xi_{j} \circ \mathcal{H}_{u}=\pi_{2} \circ\left(\mathcal{F}_{i} \times \mathcal{F}_{j} \xi_{j}\right) \circ \kappa_{u}$ by naturality of $\xi: \mathcal{H} \rightarrow \mathcal{H}^{\prime}$.

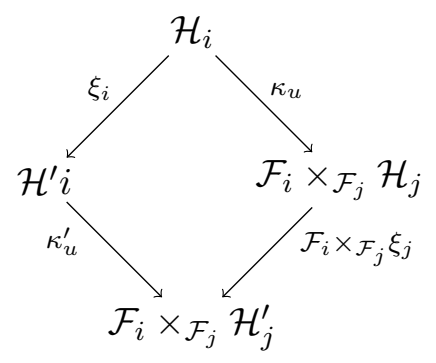

Conversely, any modification $\Xi: \kappa \rightarrow \kappa^{\prime}$ is a natural transformation $\left\{\Xi_{i}\right\}_{i \in \mathbf{J}}: \mathcal{H} \rightarrow \mathcal{H}^{\prime}$ where $\mathcal{H}$ and $\mathcal{H}^{\prime}$ are the respective carrier functors - naturality follows directly from the commutativity of the diagram above (taking $\xi_{i}=\Xi_{i}$ and $u=\mathrm{id}_{i}$ ).

Corollary 5.2. For any functor $\mathcal{F} \in[\mathbf{J}, \mathbf{C}]$, the strict homomorphism $\mathbf{\Gamma}$ defines a natural isomorphism between the following two functors of type $[\mathbf{J}, \mathbf{C}] \rightarrow \mathbf{C a t}$ :

$$
\operatorname{Span}([\mathbf{J}, \mathbf{C}])\left(\mathcal{F},{ }_{\boldsymbol{\iota}}\right) \cong \operatorname{Hom}_{l}[\mathbf{J}, \operatorname{Span}(\mathbf{C})]\left(\Gamma \mathcal{F}, \Gamma_{\boldsymbol{\iota}}\right) .
$$

The above lemma and corollary can be adapted for strong transformations instead of lax ones (this will recur when we discuss bicolimits formally). The restriction to strong transformations has a counterpart on the other side of the isomorphism of Corollary 5.2. we need to restrict to those spans in $\operatorname{Span}([\mathbf{J}, \mathbf{C}])(\mathcal{F}, \mathcal{G})$ that have a cartesian transformation from the carrier to $\mathcal{F}$.

Recall that a cartesian transformation between functors is a natural transformation with all naturality squares pullbacks (see Definition 4.1). It is an easy exercise to show that cartesian transformations include all natural isomorphisms and are closed under pullback. Hence - similarly to how one restricts the arrows of a span bicategory to partial maps i.e. those spans with the left component mono - we let $\operatorname{Span}_{[\mathbf{J}, \mathbf{C}]}^{\leftarrow}$ be the (non-full) sub-bicategory of $\operatorname{Span}([\mathbf{J}, \mathbf{C}])$ that has as arrows from $\mathcal{F}$ to $\mathcal{G}$ those spans in which the left component is cartesian. Adapting the proof of Lemma 5.1, one obtains the following.

Proposition 5.3. There is a strict homomorphism $\boldsymbol{\Gamma}: \operatorname{Span}_{[\mathbf{J}, \mathbf{C}]}^{\leftarrow} \rightarrow \operatorname{Hom}[\mathbf{J}, \operatorname{Span}(\mathbf{C})]$ which is full and faithful on both arrows and 2-cells. For any functor $\mathcal{F} \in[\mathbf{J}, \mathbf{C}], \boldsymbol{\Gamma}$ defines a natural isomorphism between the following functors $[\mathbf{J}, \mathbf{C}] \rightarrow$ Cat:

$$
\operatorname{Span}_{[\mathbf{J}, \mathbf{C}]}^{\leftarrow}\left(\mathcal{F},{ }_{\boldsymbol{L}}\right) \cong \operatorname{Hom}[\mathbf{J}, \operatorname{Span}(\mathbf{C})]\left(\Gamma \mathcal{F}, \Gamma_{\mathbf{乙}}\right)
$$

The above lets us pass between diagrams in $\operatorname{Span}(\mathbf{C})$ and $\mathbf{C}$ : for example the strong transformations of homomorphisms to $\operatorname{Span}(\mathbf{C})$ are those spans of natural transformations of functors to $\mathbf{C}$ that have a cartesian first component; the modifications of the former are the morphisms of spans of the latter. This observation will be useful when relating the notion of bicolimit in $\operatorname{Span}(\mathbf{C})$ with the notion of vK-cocone in $\mathbf{C}$.

For our purposes we need to recall only the definition of (conical) bicolimits 25] for functors with domain an (ordinary) small category $\mathbf{J}$. Given a homomorphism $\mathcal{M}: \mathbf{J} \rightarrow$ $\mathscr{B}$, a bicolimit of $\mathcal{M}$ is an object bicol $\mathcal{M} \in \mathscr{B}$ together with a pseudo-cocone $\kappa: \mathcal{M} \rightarrow$ $\Delta($ bicol $\mathcal{M})$ such that "pre-composition" with $\kappa$ gives an equivalence of categories

$$
\mathscr{B}(\operatorname{bicol} \mathcal{M}, X) \simeq \operatorname{Hom}[\mathbf{J}, \mathscr{B}](\mathcal{M}, \Delta X)
$$


that is natural in $X$ (i.e. the right hand side is essentially representable as a functor $\lambda X$. Hom $[\mathbf{J}, \mathscr{B}](\mathcal{M}, \Delta X): \mathscr{B} \rightarrow \mathbf{C a t})$; the pair $\langle$ bicol $\mathcal{M}, \kappa\rangle$ is referred to as the bicolimit of $\mathcal{M}$. We will often speak of $\kappa: \mathcal{M} \rightarrow \Delta$ bicol $\mathcal{M}$ as a bicolimit without mentioning the pair $\langle$ bicol $\mathcal{M}, \kappa\rangle$ explicitly.

To make the connection with the elementary characterisation of Van Kampen cocones in Proposition 4.3, we use the fact that equivalences of categories can be characterised as full, faithful functors that are essentially surjective on objects to derive the following equivalent, elementary definition.

Definition 5.4 (Bicolimits). Given an su-bicategory $\mathscr{B}$, a category $\mathbf{J}$ and a strict homomorphism $\mathcal{M}: \mathbf{J} \rightarrow \mathscr{B}$, a bicolimit for $\mathcal{M}$ consists of:

- an object bicol $\mathcal{M} \in \mathscr{B}$;

- a pseudo-cocone $\kappa: \mathcal{M} \rightarrow \Delta$ bicol $\mathcal{M}$ : for each $i \in \mathbf{J}$ an arrow $\kappa_{i}: \mathcal{M}_{i} \rightarrow$ bicol $\mathcal{M}$, and for each $u: i \rightarrow j$ in $\mathbf{J}$ an invertible 2 -cell $\kappa_{u}: \kappa_{i} \rightarrow \kappa_{j} \circ \mathcal{M}_{u}$ satisfying the axioms required for $\kappa$ to be a strong transformation.

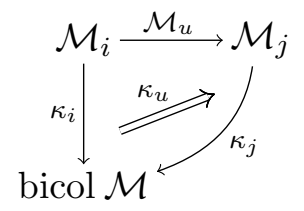

The bicolimit satisfies the following universal properties.

(i) essential surjectivity:

for any pseudo-cocone $\lambda: \mathcal{M} \rightarrow \Delta X$, there exists $h$ : bicol $\mathcal{M} \rightarrow X$ in $\mathscr{B}$ and an invertible modification $\Theta: \lambda \rightarrow \Delta h \odot \kappa$. The pair $\langle h, \Theta\rangle$ is called a mediating cell from $\kappa$ to $\lambda$.

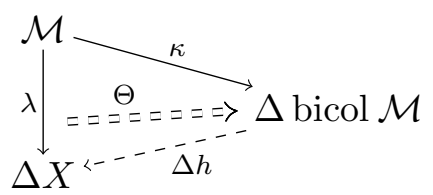

(ii) fullness and faithfulness:

for any $h, h^{\prime}$ : bicol $\mathcal{M} \rightarrow X$ in $\mathscr{B}$ and each modification $\Xi: \Delta h \odot \kappa \rightarrow \Delta h^{\prime} \odot \kappa$, there is a unique 2-cell $\xi: h \rightarrow h^{\prime}$ satisfying $\Xi=\Delta \xi * \iota_{\kappa}$ (and hence $\xi$ is invertible iff $\Xi$ is).

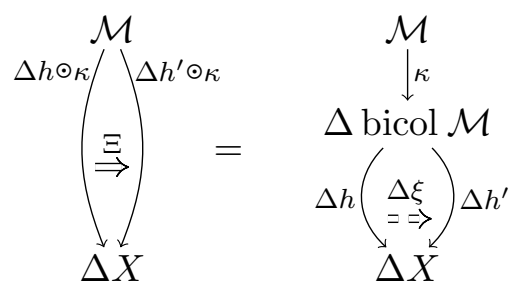

Condition (iii) of this definition implies that mediating cells from a bicolimit to a pseudococone are essentially unique: any two such mediating cells $\langle h, \Theta\rangle$ and $\left\langle h^{\prime}, \Theta^{\prime}\right\rangle$ are isomorphic since $\Theta^{\prime} \odot \Theta^{-1}: \Delta h \odot \kappa \rightarrow \Delta h^{\prime} \odot \kappa$ corresponds to a unique invertible 2-cell $\zeta: h \rightarrow h^{\prime}$ such that $\Theta^{\prime} \odot \Theta^{-1}=\Delta \zeta * I_{\kappa}$.

To facilitate the exposition of the relationship between the bicolimits in $\operatorname{Span}(\mathbf{C})$ and VK-cocones in $\mathbf{C}$ we shall first reformulate the above elementary definition of bicolimits. Given a pseudo-cocone $\kappa: \mathcal{M} \rightarrow \Delta C$, a morphism $h: C \rightarrow D$ will be called universal for $\kappa$ or $\kappa$-universal if, given any other morphism $h^{\prime}: C \rightarrow D$ with a modification $\Xi: \Delta h \odot \kappa \rightarrow \Delta h^{\prime} \odot \kappa$, 
there exists a unique 2-cell $\xi: h \rightarrow h^{\prime}$ satisfying $\Xi=\Delta \xi * I_{\kappa}$; further, a mediating cell $\langle h, \Theta\rangle$ is called universal, if the morphism $h$ is universal. The motivation behind this terminology and the slightly redundant statement of the following proposition will become apparent in Section 6, its proof is straightforward.

Proposition 5.5. A pseudo-cocone $\kappa: \mathcal{M} \rightarrow \Delta C$ from a diagram $\mathcal{M}$ to $C$ is a bicolimit iff both of the following hold:

(i) for any pseudo cocone $\lambda: \mathcal{M} \rightarrow \Delta D$ there is a universal mediating cell $\langle h: C \rightarrow D, \Theta\rangle$ from $\kappa$ to $\lambda$;

(ii) all arrows $h: C \rightarrow D$ are universal for $\kappa$.

We are interested in bicolimits of strict homomorphisms of the form $\Gamma \mathcal{F}$ where $\mathcal{F}: \mathbf{J} \rightarrow$ $\mathbf{C}$ is a functor and $\Gamma: \mathbf{C} \rightarrow \operatorname{Span}(\mathbf{C})$ is the covariant embedding of $\mathbf{C}$. The defining equivalence of bicolimits in (5.1) specialises as follows:

$$
\operatorname{Span}(\mathbf{C})(\operatorname{bicol} \Gamma \mathcal{F}, X) \simeq \operatorname{Hom}[\mathbf{J}, \operatorname{Span}(\mathbf{C})](\Gamma \mathcal{F}, \Delta X) .
$$

Using Proposition 5.3, this is equivalent to:

$$
\operatorname{Span}(\mathbf{C})(\operatorname{bicol} \Gamma \mathcal{F}, X) \simeq \operatorname{Span}_{[\mathbf{J}, \mathbf{C}]}^{\leftarrow}(\mathcal{F}, \Delta X)
$$

We shall exploit working in $\operatorname{Span}_{[\mathbf{J}, \mathbf{C}]}^{\leftarrow}$ in the following lemma which relates the concepts involved in the elementary definition of bicolimits with diagrams in $\mathbf{C}$. It will serve as the technical backbone of our main theorem.

Lemma 5.6 (Mediating cells and universality for spans). Let $\kappa: \mathcal{F} \rightarrow \Delta C$ be a cocone in $\mathbf{C}$ of a diagram $\mathcal{F} \in[\mathbf{J}, \mathbf{C}]$, and let $\lambda: \Gamma \mathcal{F} \rightarrow \Delta D$ be a pseudo-cocone in $\operatorname{Span}(\mathbf{C})$ where $\lambda_{i}=\left(\varphi_{i}, \psi_{i}\right)$ for all $i \in \mathbf{J}$ :

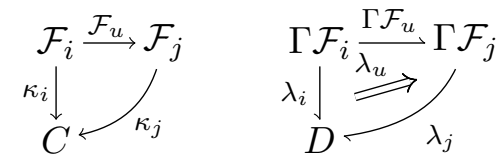

(i) to give a mediating cell

$$
\left\langle C \stackrel{h_{1}}{\longleftarrow} H \stackrel{h_{2}}{\longrightarrow} D, \Theta: \lambda \rightarrow \Delta\left(h_{1}, h_{2}\right) \odot \Gamma \kappa\right\rangle
$$

from $\Gamma \kappa$ to $\lambda$ is to give a cocone $\vartheta: \mathcal{H} \rightarrow \Delta H$ where $\mathcal{H}$ is the carrier functor of the image of $\lambda$ in $\operatorname{Span}_{[\mathbf{J}, \mathbf{C}]}^{\leftarrow}(\mathcal{F}, \Delta D)$ (see Proposition $[5.3)$ such that the resulting threedimensional diagram $(\dagger)$ in $\mathbf{C}$ (below) commutes and its lateral faces $\underset{\mathcal{F}_{i}}{\mathcal{H}_{i}} \underset{\rightarrow}{\rightarrow} \downarrow_{C}^{H}$ are pullbacks;

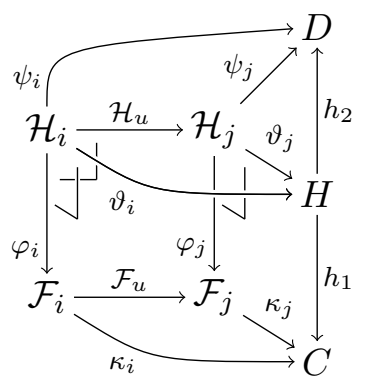


(ii) to give a modification $\Xi: \Delta\left(h_{1}, h_{2}\right) \odot \Gamma \kappa \rightarrow \Delta\left(h_{1}^{\prime}, h_{2}^{\prime}\right) \odot \Gamma \kappa$ for a pair of spans

$$
\left(h_{1}, h_{2}\right),\left(h_{1}^{\prime}, h_{2}^{\prime}\right): C \rightarrow D
$$

is to give a cartesian transformation $\Xi: \mathcal{F} \times_{\Delta C} \Delta H \rightarrow \mathcal{F} \times_{\Delta C} \Delta H^{\prime}$ such that the two equations $\pi_{1}^{\prime} \circ \Xi=\pi_{1}$ and $\left(\Delta h_{2}^{\prime}\right) \circ \pi_{2}^{\prime} \circ \Xi=\left(\Delta h_{2}\right) \circ \pi_{2}$ hold.
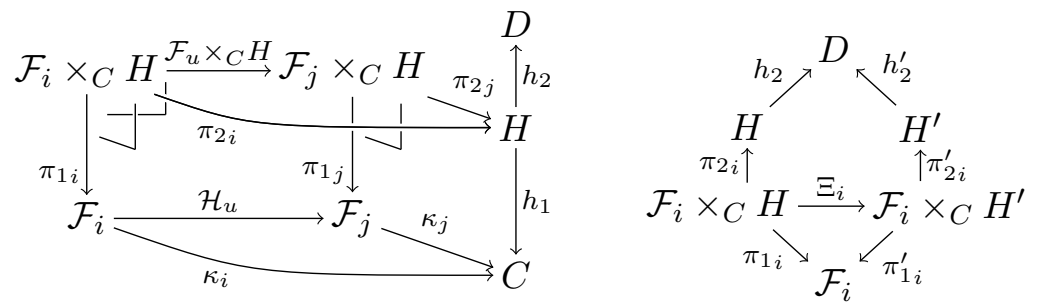

Here $\mathcal{F} \leftarrow \pi_{1}-\mathcal{F} \times_{\Delta C} \Delta H-\pi_{2} \rightarrow \Delta H$ is the pullback of $\mathcal{F}-\kappa \rightarrow \Delta C \leftarrow \Delta h_{1}-\Delta H$ as sketched in $(\ddagger)$ above and similarly for $h^{\prime}: H^{\prime} \rightarrow C$. Further, to give a cell $\xi:\left(h_{1}, h_{2}\right) \rightarrow\left(h_{1}^{\prime}, h_{2}^{\prime}\right)$ that satisfies $\Delta \xi * I_{\Gamma \kappa}=\Xi$ is to give a $\mathbf{C}$-arrow $\xi: H \rightarrow H^{\prime}$ which satisfies the three equations $h_{1}^{\prime} \circ \xi=h_{1}, h_{2}^{\prime} \circ \xi=h_{2}$ and $\Delta \xi \circ \pi_{2}=\pi_{2}^{\prime} \circ \Xi$;

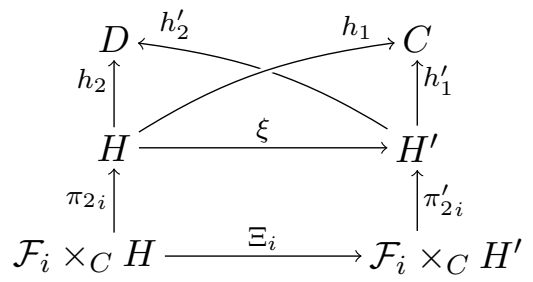

(iii) given a span $\left(h_{1}, h_{2}\right): C \rightarrow D$, if the pullback of $\kappa$ along $h_{1}$ is a colimit, i.e. if $\pi_{2}: \mathcal{F} \times_{\Delta C} \Delta H \rightarrow \Delta H$ is a colimit, then $\left(h_{1}, h_{2}\right)$ is universal for $\Gamma \kappa$;

(iv) conversely, if $\left(h_{1}, h_{2}\right)$ is universal for $\Gamma \kappa$, then $\pi_{2}: \mathcal{F} \times_{\Delta C} \Delta H \rightarrow \Delta H$ is a colimitprovided that some colimit of $\mathcal{F} \times \Delta C \Delta H$ exists in $\mathbf{C}$.

Proof. Both (ii) and (iii) are immediate consequences of Proposition 5.3 .

As for (iii), we need to show that every modification $\Xi: \Delta\left(h_{1}, h_{2}\right) \odot \Gamma \kappa \rightarrow \Delta\left(h_{1}^{\prime}, h_{2}^{\prime}\right) \odot \Gamma \kappa$ is equal to $\Delta \xi * I_{\Gamma \kappa}$ for a unique $\xi:\left(h_{1}, h_{2}\right) \rightarrow\left(h_{1}^{\prime}, h_{2}^{\prime}\right)$. By (iii), $\Xi$ is a natural transformation $\Xi: \mathcal{F} \times{ }_{\Delta C} \Delta H \rightarrow \mathcal{F} \times{ }_{\Delta C} \Delta H^{\prime}$. Then, by naturality of $\Xi$, we have that $\pi_{2 j}^{\prime} \circ \Xi_{j} \circ\left(\mathcal{F}_{u} \times{ }_{\Delta C}\right.$ $\Delta H)=\pi_{2 i}^{\prime} \circ \Xi_{i}$ holds for all $u: i \rightarrow j$ in $\mathbf{J}$, and since $\pi_{2}$ is a colimit we have a unique $\xi: H \rightarrow H^{\prime}$ satisfying $\xi \circ \pi_{2 i}=\pi_{2 i}^{\prime} \circ \Xi_{i}$ for all $i \in \mathbf{J}$. The equations $h_{i}=h_{i}^{\prime} \circ \xi$ follow from the universal property of $\pi_{2}$ (and the properties of $\Xi$ ). To show uniqueness of $\xi$, let $\zeta:\left(h_{1}, h_{2}\right) \rightarrow\left(h_{1}^{\prime}, h_{2}^{\prime}\right)$ be a 2 -cell such that $\Xi=\Delta \zeta * I_{\Gamma_{\kappa}}$; then using the second statement of Lemma 5.6(iii), $\Delta \zeta \circ \pi_{2}=\pi_{2}^{\prime} \circ \Xi$; hence $\zeta=\xi$ follows since $\pi_{2}$ is a colimit. In summary, $\left(h_{1}, h_{2}\right)$ is universal for $\Gamma \kappa$.

To show (iv), let $\left\langle H^{\prime}, \vartheta\right\rangle$ be a colimit of $\mathcal{F} \times{ }_{\Delta C} \Delta H$. Now, it suffices to show that there is a $\mathbf{C}$-morphism $\xi: H \rightarrow H^{\prime}$ such that $\vartheta=\Delta \xi \circ \pi_{2}$. By the universal property of $\vartheta$, we obtain unique C-arrows $h_{1}^{\prime}: H^{\prime} \rightarrow C$ and $h_{2}^{\prime}: H^{\prime} \rightarrow D$ such that $\Delta h_{1}^{\prime} \circ \vartheta=\kappa \circ \pi_{1}$ and $\Delta h_{2}^{\prime} \circ \vartheta=\Delta h_{2} \circ \pi_{2}$. It also follows that the two equations $h_{1} \circ k=h_{1}^{\prime}$ and $h_{2} \circ k=h_{2}^{\prime}$ hold. Pulling back $\kappa$ along $h_{1}^{\prime}$ yields a span $\mathcal{F} \leftarrow \pi_{1}^{\prime}-\mathcal{F} \times \Delta C \Delta H^{\prime}-\pi_{2}^{\prime} \rightarrow \Delta H^{\prime}$; we then obtain a natural transformation $\Xi: \mathcal{F} \times_{\Delta C} \Delta H \rightarrow \mathcal{F} \times \Delta C \Delta H^{\prime}$ which satisfies $\pi_{1}=\pi_{1}^{\prime} \circ \Xi$ and $\vartheta=\pi_{2}^{\prime} \circ \Xi$,

\footnotetext{
${ }^{2}$ The reason is that once such a $\xi$ is provided, there is a unique $k: H^{\prime} \rightarrow H$ satisfying $\Delta k \circ \vartheta=\pi_{2}$, and thus $\xi \circ k=\mathrm{id}_{H^{\prime}}$ by the universal property of colimits; moreover $k \circ \xi=\mathrm{id}_{H}$ must hold since $\left(h_{1}, h_{2}\right)$ is universal for $\Gamma \kappa$.
} 
and hence also $\Delta h_{2} \circ \pi_{2}=\Delta h_{2}^{\prime} \circ \vartheta=\Delta h_{2}^{\prime} \circ \pi_{2}^{\prime} \circ \Xi$. By (iii), this defines a modification $\Xi: \Delta\left(h_{1}, h_{2}\right) \odot \Gamma \kappa \rightarrow \Delta\left(h_{1}^{\prime}, h_{2}^{\prime}\right) \odot \kappa$. Using universality, we get a unique $\xi: H \rightarrow H^{\prime}$ such that $h_{1}^{\prime} \circ \xi=h_{1}, h_{2}^{\prime} \circ \xi=h_{2}$ and $\Delta \xi \circ \pi_{2}=\pi_{2}^{\prime} \circ \Xi=\vartheta$.

\section{VAN Kampen COCONES aS SPAN BICOLIMits}

Here we prove the main result of this paper, Theorem 6.3. Roughly speaking, the conclusion is that (under natural assumptions - existence of pullbacks and enough colimits in $\mathbf{C}$ ) to be VK in $\mathbf{C}$ is to be a bicolimit in $\operatorname{Span}(\mathbf{C})$. The consequence is that "being VK" is a universal property - in $\operatorname{Span}(\mathbf{C})$ rather than in $\mathbf{C}$.

The proof relies on a correspondence between the elementary characterisation of Van Kampen cocones in $\mathbf{C}$ of Proposition 4.3 and the universal properties of pseudo-cocones in $\operatorname{Span}(\mathbf{C})$ of Proposition 5.5. More precisely, given a colimit $\kappa: \mathcal{M} \rightarrow \Delta C$ in $\mathbf{C}$, we shall show that:

- $\Gamma \kappa$-universality of all spans $\left(h_{1}, h_{2}\right): C \rightarrow D$ corresponds to the implication (ii) $\Rightarrow$ (i) of Proposition 4.3, which is also known as pullback-stability or universality of the colimit $\kappa$;

- existence of some universal mediating cell from $\Gamma \kappa$ to any $\lambda: \Gamma \kappa \rightarrow \Delta D$ is the counterpart of the implication (i) $\Rightarrow$ (ii) of Proposition 4.3, which-for want of a better name-we here refer to as "converse universality" of $\kappa$;

- thus, $\Gamma \kappa$ is a bicolimit in $\operatorname{Span}(\mathbf{C})$ if and only if the colimit $\kappa$ is Van Kampen.

The first two points are made precise by the statements of the following two lemmas. The third point is the statement of the main theorem.

Lemma 6.1 (Converse universality). Let $\mathcal{F} \in[\mathbf{J}, \mathbf{C}]$ where $\mathbf{C}$ has pullbacks and for all $(\tau: \mathcal{E} \rightarrow \mathcal{F}) \in[\mathbf{J}, \mathbf{C}] \Downarrow \mathcal{F}$ a colimit of $\mathcal{E}$ exists. Then $\kappa: \mathcal{F} \rightarrow \Delta C$ satisfies "converse universality" iff given any pseudo-cocone $\lambda: \Gamma \mathcal{F} \rightarrow \Delta D$, there exists a universal mediating cell $\left\langle\left(h_{1}, h_{2}\right), \Theta\right\rangle$ from $\Gamma \kappa$ to $\lambda$ in $\operatorname{Span}(\mathbf{C})$.

Proof. $(\Rightarrow)$ Suppose that $\lambda: \Gamma \mathcal{F} \rightarrow \Delta D$ is a pseudo-cocone in $\operatorname{Span}(\mathbf{C})$.

For $u: i \rightarrow j$ in $\mathbf{J}$, we obtain a commutative diagram, as illustrated (see Proposition [5.3). Let $\vartheta: \mathcal{H} \rightarrow \Delta H$ be the colimit of $\mathcal{H}$; thus we obtain $h_{1}: H \rightarrow C$ and $h_{2}: H \rightarrow D$ making diagram (†) commute. By converse universality, the side faces ${\underset{\mathcal{F}}{\mathcal{F}_{i}}}_{i} \downarrow_{\rightarrow} \downarrow_{C}^{H}$ are pullback squares; using Lemma 5.6(iii) we get an invertible modification $\Theta: \lambda \rightarrow \Delta\left(h_{1}, h_{2}\right) \odot \Gamma \kappa$. That $\left(h_{1}, h_{2}\right)$ is universal follows from Lemma 5.6(iii) since $\vartheta$ is a colimit.

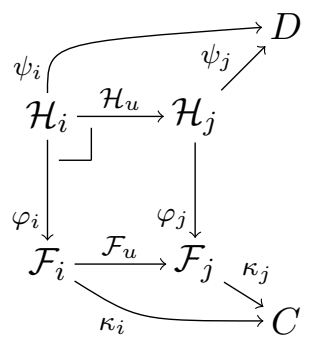

$(\Leftarrow)$ If in diagram $(\dagger) D=\operatorname{col} \mathcal{H}$ and $\langle D, \psi\rangle$ is the corresponding colimit, we first use the assumption to obtain a universal mediating cell $\left\langle\left(h_{1}, h_{2}\right), \Theta\right\rangle$ from $\Gamma \kappa$ to $\lambda^{(\varphi, \psi)}$ where $\lambda^{(\varphi, \psi)}$ is the pseudo-cocone corresponding to the cartesian transformations $\varphi: \mathcal{H} \rightarrow \mathcal{F}$ 
and $\psi: \mathcal{H} \rightarrow \Delta D$ such that $\lambda_{i}^{(\varphi, \psi)}=\left(\varphi_{i}, \psi_{i}\right)$ as in Lemma 5.6(1i); the latter also provides $\vartheta: \mathcal{H} \rightarrow \Delta H$ such that $h_{2} \circ \vartheta_{i}=\psi_{i}$ and all $\mathcal{F}_{i} \stackrel{\mathcal{H}}{\rightarrow}_{\rightarrow}^{\rightarrow} \downarrow_{C}^{H}$ are pullback squares.

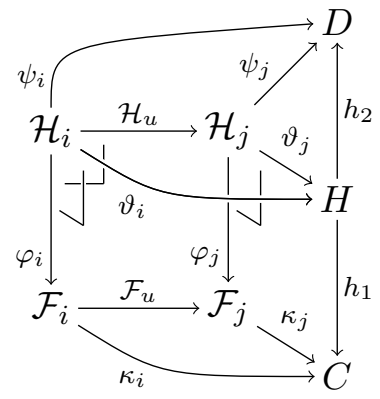

It suffices to show that $h_{2}=\operatorname{id}_{H}$. However, by the universal property of the colimit $\langle D, \psi\rangle$, there is an arrow $k: D \rightarrow h$ such that $k \circ \psi_{i}=\vartheta_{i}$. The equation $h_{2} \circ k=\mathrm{id}_{D}$ holds because $\langle D, \psi\rangle$ is a colimit in $\mathbf{C}$, and $k \circ h_{2}=\mathrm{id}_{H}$ follows since $\left(h_{1}, h_{2}\right)$ is universal for $\Gamma \kappa$.

Lemma 6.2 (Universality). Consider $\mathcal{F} \in[\mathbf{J}, \mathbf{C}]$ where $\mathbf{C}$ has pullbacks such that for all $(\tau: \mathcal{E} \rightarrow \mathcal{F}) \in[\mathbf{J}, \mathbf{C}] \Downarrow \mathcal{F}$, a colimit of $\mathcal{E}$ exists. Then $\kappa: \mathcal{F} \rightarrow \Delta C$ satisfies universality iff every morphism $\left(h_{1}, h_{2}\right): C \rightarrow D$ in $\operatorname{Span}(\mathbf{C})$ is universal for $\Gamma \kappa$.

Proof. $(\Rightarrow)$ Any morphism $\left(h_{1}, h_{2}\right)$ leads to a diagram $(\ddagger)$ where all the side-faces are pullbacks. By universality of $\kappa$, the cocone $\pi_{2}$ of the top face is a colimit; thus $\left(h_{1}, h_{2}\right)$ is universal for $\Gamma \kappa$ by Lemma 5.6(iii).

$(\Leftarrow)$ Suppose that in diagram $(\ddagger)$ the side faces are all pullbacks. By assumption $\left(h_{1}, h_{2}\right)$ is universal for $\Gamma \kappa$, thus $\left\langle H, \pi_{2}: \mathcal{F} \times_{\Delta C} \Delta H \rightarrow \Delta H\right\rangle$ is a colimit by Lemma 5.6(iv).

Finally, these two lemmas together with Proposition [5.5] imply our main result.

Theorem 6.3. Let $\mathcal{F} \in[\mathbf{J}, \mathbf{C}]$ where $\mathbf{C}$ has pullbacks and for all cartesian transformations $\tau: \mathcal{E} \rightarrow \mathcal{F}$, a colimit of $\mathcal{E}$ exists. Then a cocone $\kappa: \mathcal{F} \rightarrow \Delta C$ is Van Kampen iff $\Gamma \kappa: \Gamma \mathcal{F} \rightarrow$ $\Delta C$ is a bicolimit in $\operatorname{Span}(\mathbf{C})$.

\section{Conclusion, Related WORK And Future WORK}

We gave a general definition of Van Kampen cocone that captures several previously studied notions in computer science, topology, and related areas, showing that they are instances of the same concept. Moreover, we have provided two alternative characterisations: the first one is elementary, and involves only basic category theoretic notions; the second one exhibits it as a universal property: Van Kampen cocones are just those colimits that are preserved by the canonical covariant embedding into the span bicategory.

There is some interesting related recent work. Milius [33] showed that coproducts are preserved (as a lax-adjoint-cooplimit) in the 2-category of relations over an extensive category C. Cockett and Guo [9] have investigated the general conditions under which partial map categories are join-restriction categories: roughly, certain colimits in the underlying category are required to be VK-cocones.

Finally, the definition of Van Kampen cocone allows for several natural variations. For example, one may replace the slice category over the object at the "tip" of cocones by a (full) subcategory of it; this is exactly the step from global descent to $\mathbb{E}$-descent [19] and is closely related to the proposals in [11, 13, for a weakening of the notion of adhesivity. 
Alternatively, one may start with cocones or diagrams of a particular form. In this way quasi-adhesive categories [29] arise as in the latter only pushouts along regular monos are required to be VK; another example is the work of Cockett and Guo [9], where Van Kampen cocones exist for a class of diagrams that naturally arises in their study of join restriction categories. Thus, possibly combining the latter two ideas, several new forms of Van Kampen cocones and diagrams arise as the subject for future research.

\section{REFERENCES}

[1] S. Abramsky. Interaction categories. In Theory and Formal Methods '93, Workshops in Computing, pages 57-69. Springer, 1993.

[2] P. Aczel and N. Mendler. A final coalgebra theorem. In Category Theory and Computer Science (CTCS '89), volume 389 of $L N C S$, pages 357-365. Springer, 1989.

[3] M. Barr. Exact categories. In Exact Categories and Categories of Sheaves, volume 236 of LNM, pages 1-120. Springer, 1971.

[4] J. Bénabou. Introduction to bicategories, part 1. In Midwest Category Seminar, volume 47 of LNM, pages 1-77. Springer, 1967.

[5] F. Borceux. Handbook of categorical algebra, volume 1. Cambridge University Press, 1994.

[6] R. Brown and G. Janelidze. Van Kampen theorems for categories of covering morphisms in lextensive categories. J. Pure Appl. Algebra, 119:255-263, 1997.

[7] A. Carboni, S. Lack, and R. F. C. Walters. Introduction to extensive and distributive categories. J. Pure Appl. Algebra, 84:145-158, February 1993.

[8] J. R. B. Cockett and D. A. Spooner. Constructing process categories. Theor. Comput. Sci., 177(1):73109, 1997.

[9] R. Cockett and X. Guo. Join restriction categories and the importance of being adhesive. Unpublished manuscript, 2007.

[10] A. Corradini, U. Montanari, F. Rossi, H. Ehrig, R. Heckel, and M. Löwe. Algebraic approaches to graph transformation - part I: Basic concepts and double pushout approach. In Handbook of Graph Grammars, pages 163-246. World Scientific, 1997.

[11] H. Ehrig, A. Habel, J. Padberg, and U. Prange. Adhesive high-level replacement categories and systems. In Graph Transformation (ICGT '04), volume 3256 of LNCS, pages 144-160. Springer, 2004.

[12] H. Ehrig and B. König. Deriving bisimulation congruences in the DPO approach to graph rewriting. In Foundations of Software Science and Computation Structures (FoSSaCS '04), LNCS. Springer, 2004.

[13] H. Ehrig and U. Prange. Weak adhesive high-level replacement categories and systems: a unifying framework for graph and Petri net transformations. In Essays dedicated to Joseph A. Goguen, volume 2987 of $L N C S$, pages 235-251. Springer, 2006.

[14] P. J. Freyd and A. Scedrov. Categories, allegories. North-Holland, 1990.

[15] F. Gadducci, R. Heckel, and M. Llabrés. A bi-categorical axiomatisation of concurrent graph rewriting. In Category Theory and Computer Science (CTCS ‘99), volume 29 of ENTCS. Elsevier, 1999.

[16] T. Heindel. Hereditary pushouts reconsidered. In Graph Transformation (ICGT '10), volume 6372 of LNCS, pages 250-265. Springer, 2010. To appear.

[17] T. Heindel and P. Sobociński. Van Kampen colimits as bicolimits in Span. In Algebra and Coalgebra in Computer Science (CALCO '09), number 5728 in LNCS, pages 335-349. Springer, 2009.

[18] B. Jacobs. Parameters and parametrization in specification, using distributive categories. Fundam. Inform., 24(3):209-250, 1995.

[19] G. Janelidze and W. Tholen. Facets of descent, I. Appl. Categor. Struct., 2(3):245-281, 1994.

[20] P. T. Johnstone. Topos theory, volume 10 of L. M. S. Monographs. Academic Press, 1977.

[21] P. T. Johnstone. Sketches of an elephant: a topos theory compendium, volume 1. Clarendon Press, 2002.

[22] P. T. Johnstone, S. Lack, and P. Sobociński. Quasitoposes, quasiadhesive categories and Artin glueing. In Algebra and Coalgebra in Computer Science (CALCO '07), volume 4626 of LNCS, pages 312-326. Springer, 2007.

[23] A. Joyal, M. Nielsen, and G. Winsk. Bisimulation from open maps. Inf. Comput., 127:164-185, 1994. 
[24] P. Katis, N. Sabadini, and R. F. C. Walters. Span(Graph): an algebra of transition systems. In Algebraic Methodology and Software Technology (AMAST '97), volume 1349 of LNCS, pages 322-336. Springer, 1997.

[25] G. M. Kelly. Elementary observations on 2-categorical limits. Bull. Austral. Math. Soc., 39:301-317, 1989.

[26] G. M. Kelly and R. H. Street. Review of the elements of 2-categories. In Category Theory, number 420 in LNM, pages 75-103. Springer, 1973.

[27] S. Lack. A 2-categories companion. In Towards Higher Categories. Institute for Mathematics and its Applications, 2010. To appear.

[28] S. Lack and P. Sobociński. Adhesive categories. In Foundations of Software Science and Computation Structures (FoSSaCS '04), volume 2987 of LNCS, pages 273-288. Springer, 2004.

[29] S. Lack and P. Sobociński. Adhesive and quasiadhesive categories. RAIRO-Theor. Inf. Appl., 39(3):511$546,2005$.

[30] S. Lack and P. Sobociński. Toposes are adhesive. In Graph Transformation (ICGT '06), volume 4178 of LNCS, pages 184-198. Springer, 2006.

[31] F. W. Lawvere. Some thoughts on the future of category theory. In Category Theory (CT '91), volume 1488 of $L N M$. Springer, 1991.

[32] H. Lindner. A remark on Mackey-functors. Manuscripta Math., 18(3):273-278, 1976.

[33] S. Milius. On colimits in categories of relations. Appl. Categor. Struct., 11(3):287-312, 2003.

[34] V. Sassone and P. Sobociński. Reactive systems over cospans. In Logic in Computer Science (LiCS '05), pages 311-320. IEEE Press, 2005.

[35] D. Scott. Continuous lattices. In Toposes, algebraic geometry and logic, volume 274 of $L N M$, pages 97-136. Springer, 1972.

[36] R. F. C. Walters. Categories and Computer Science. Carslaw Publications, 1991.

[37] G. Winskel and M. Nielsen. Models for concurrency. In S. Abramsky, D. Gabbay, and T. Maibaum, editors, Handbook of Logic in Computer Science, pages 1-148. Oxford University Press, 1995.

[38] O. Wyler. Lecture notes on topoi and quasitopoi. World Scientific, 1991. 